\title{
STATIC SELF-GRAVITATING ELASTIC BODIES IN EINSTEIN GRAVITY
}

\author{
LARS ANDERSSON, ROBERT BEIG, AND BERND G. SCHMIDT
}

\begin{abstract}
We prove that given a stress-free elastic body there exists, for sufficiently small values of the gravitational constant, a unique static solution of the Einstein equations coupled to the equations of relativistic elasticity. The solution constructed is a small deformation of the relaxed configuration. This result yields the first proof of existence of static solutions of the Einstein equations without symmetries.
\end{abstract}

\section{INTRODUCTION}

General relativistic effects generated by compact, isolated bodies, such as stars and even satellites, are of increasing importance in observational astronomy and experimental general relativity. Considering this fact, it is remarkable how little is known about solutions of the Einstein field equations for systems with spatially compact sources. The situation is not much better if we describe gravity by Newton's theory.

The present paper provides the first existence result for compact, isolated, static elastic bodies in Einstein's theory of gravity. With the notable exception of collisionless matter, essentially all previous results concerning compact, isolated, self-gravitating bodies deal with static or stationary fluid bodies. Under reasonable conditions, static fluid bodies are spherically symmetric, while stationary fluid bodies are axi-symmetric. Although the variational formulation of elasticity has strong similarities with that of fluid models, static elastic bodies may, in contrast to static fluid bodies, be non-symmetric. In fact, in this paper we prove, for the first time, existence of static solutions of the Einstein equations without symmetries.

1.1. Compact bodies. Fluids and dust (i.e. a pressure-less fluid) are the conceptually simplest and most commonly used matter models. For a self-gravitating compact body, it is necessary to consider a free boundary problem with zero traction on the boundary. It was only recently that an existence proof was given by Lindblad for the Cauchy problem for a nonrelativistic perfect fluid with free boundary, in the absence of gravity [24, 23]. For self-gravitating fluids, no results of this generality are known, and it is only in the static or stationary cases that results are available.

If we assume that spacetime is static, the standard conjecture is that any isolated selfgravitating body, consisting of a perfect fluid, is spherically symmetric. This is known to be the case in Newton's theory, for a general equation of state. In Einstein's theory there is the work by Beig and Simon [8] which solves the problem for a large class equation of states; a proof in the general case remains to be found. For the case of stationary spacetimes in Einstein gravity, i.e. spacetimes with a timelike, non-hypersurface orthogonal Killing field, it is known under certain additional assumptions on the thermodynamic properties of the fluid that there exists an additional, rotational Killing field, so that stationary spacetimes containing fluid bodies are axisymmetric [25].

Date: November 20, 2006. 
In spite of the symmetry restrictions discussed above, there are rich classes of stationary and static solutions describing isolated bodies, even in Newtonian gravity. The almost completely forgotten work of Leon Lichtenstein from roughly the period 1910-1933, provides existence results in the Newtonian case for rotating fluid solutions in various configurations, see the book [22]. Inspired by Lichtenstein, Uwe Heilig showed in 1995 the existence of stationary rotating fluid solutions in Einstein's theory [15].

These results allow one to construct stationary fluid solutions with slowly rotating almost spherical balls. Further, one has rings, rings around balls and families of nested rings. It seems almost impossible to get an overview on all possibilities. New solutions can often be constructed as perturbations of known solutions. For example, starting with a static, spherically symmetric fluid ball whose existence can be shown by using ODE techiques, one may prove the existence of a rotating solution with small angular velocity. This is essentially what was done by Lichtenstein and Heilig.

The Vlasov matter model is a statistical description of weakly interacting particles. It is conceptually more difficult to work with than fluids but has been used very successfully in various circumstances. For a survey of known results, see [28]. The existence of various dynamical and time independent solution has been demonstrated. All the known stationary and static solutions have axial symmetry.

1.2. Elasticity and relativity. Elasticity is of course one of the oldest topics of theoretical physics, with origins that can be traced back to the $17^{\prime}$ th century. The book by Marsden and Hughes [26] gives a modern treatment of elasticity. Already in 1911, Herglotz [16] gave a formulation of elasticity in special relativity. There are various formulations of elasticity in the framework of general relativity, see for example Rayner [27], Carter and Quintana [11], Kijowski and Magli [18, 19], Christodoulou [12, 13], to name just a few important works. Strangely enough the problem of existence of static or dynamical selfgravitating elastic bodies in Einstein's or Newton's theory of gravity has, to the best of our knowledge, until recently not been considered. The only exception is for the spherically symmetric case. Even in non-relativistic elasticity quite little is known. The first existence theorem in three dimensional static elasticity was given by Stoppelli in 1954 [29].

About six years ago, two of the present authors (R.B. and B.S.), motivated by this state of affairs, initiated a program to develop existence results for elasticity in the setting of Einstein's theory of gravity. We first showed the existence of static solutions describing elastic bodies deformed by their own Newtonian gravitational field [5], and later established the existence of relativistic elastic bodies deformed under rigid rotation [6]. For time dependent solutions local in time and space (no boundary conditions) uniqueness was proved in [13], and for existence see [4]. In [9] an existence theorem for the motion of a free elastic body in special relativity is given.

Elasticity can be described as a Lagrangian field theory [4, 13], and hence the action for self-gravitating elastic bodies is derived by simply adding the gravitational Lagrangian to the Lagrangian for elasticity. The basic matter field is a map, the configuration, from a region in spacetime to the body, an abstract 3-manifold whose points label the constituents of the elastic body moving in spacetime. The stress of a configuration is determined by the stored energy function which completely fixes the matter model. This formulation can be used in a non relativistic spacetime, in special relativity and in Einstein's theory. Elastic materials where the stress is determined by a stored energy function are usually called hyperelastic. Diffeomorphism invariance, a necessary condition in Einstein's theory, implies that the stored energy function satisfies the additional condition known in the nonrelativistic case as material frame indifference. 
Once the stored energy function is given, the variational problem as well as the EulerLagrange equations are determined. In particular, one is led to consider Einstein's field equations with an energy momentum tensor which is determined by the deformation. The elasticity equations are a consequence of the conservation law in Einstein's theory.

As the configuration is a map from spacetime to the body, we have a free boundary value problem. To deal with this difficulty, one reformulates elasticity using deformations, i.e. maps from body to spacetime, as the basic variable. In this setting, known as the material picture, one has a fixed apriori known boundary.

Let us now consider the static self-gravitating bodies in general relativity. In this case, the theory can be given a a variational formulation on the quotient space of the timelike Killing field. Thus, in order to construct a static self-gravitating body in Einstein gravity we start from a relaxed elastic body without gravitational field and determine the deformation of such a body under its own gravitational field for small values of the gravitational constant $G$. To do this, it is convenient to choose a stored energy function for which there exists a configuration which is stress free, i.e. which satisfies, together with the Minkowski metric, the Einstein field equations for $G=0$, as well as the the elasticity equation. We start from this background solution and construct nearby self-gravitating solutions for small values of $G$.

As mentioned above, self-gravitating static fluid bodies in general relativity are known to be spherically symmetric for a large class of equations of state. In fact, the result proved in this paper provides the first example of a static solution to Einstein's field equations which is not spherically symmetric.

It is worth pointing out that the approach used in this paper cannot be applied to the problem of constructing for example an elastic neutron star since in this case, there is no nearby stress free configuration. To deal with this problem one would have to choose a stored energy function in which the shear strains are much smaller then the hydrostatic compression. Then one could begin with a spherically symmetric solution in which the radial pressure would be different from the tangential pressure. One could then use the methods developed in this paper to construct nearby solutions which are not spherically symmetric.

Finally we remark that it may be argued that the result proved in this paper is very weak, since $G$ is required to be sufficiently small. However, it should be noted that we make no restriction on the shape of the body. For example, one may consider two very large bodies connected by a very thin neck. In this situation it is clear that if we make gravity too strong (i.e. $G$ too large), the neck will break and hence there can be no static solution for such a configuration for arbitrarily large values of $G$. Thus, without restrictions on the shape of the undeformed body we can not expect a stronger result.

1.3. Overview of this paper. In section 2 we give some analytical preliminaries. We also for the convenience of the reader review some basic ideas from linearized elasticity which we will make use of. Further, we prove some results which will be used concerning Bianchi identities for weakly differentiable metrics, and concerning the divergence of tensors wich compact support. Section 3 presents the gravitational field equations in space and the elastic equations on the body. We use harmonic gauge to make the field equations elliptic. An important step is to extend the body to $\mathbb{R}^{3}$, the extended body, and to extend the deformation, the inverse configuration, to a map from the extended body to physical space, which in our case also is $\mathbb{R}^{3}$. Then we move the field equations from space to the extended body. In this way we obtain a quasilinear system of partial differential equations, the reduced 
Einstein equations in material form, were the geometrical unknowns are defined on the extended body and the elastic variables on the body.

In section 4 we formulate the reduced Einstein equations in terms of a non-linear mapping between Sobolev spaces and calculate the Frechet derivative of the map at the relaxed configuration. It contains esssentially linearized gravity and linearized elasticity. The linearized operator is Fredholm with non trivial kernel and range. The geometric reason for this is the combination of diffeomorphism invariance of the Einstein equations and Euclidean invariance of the background solution. Using an approach to some extent inspired by [21], we define a projection operator such that we can use the implicit function theorem. This way we obtain for small $G$ a solution of the reduced field equation together with the projected elasticity equations.

Section 5], which is the heart of the paper, contains a proof that the solution to the reduced, projected system obtained using the implicit function theorem, is in fact a solution of the full system of equations for the self-gravitating elastic body. At first it might seem that there are two possibilities to prove this. On the one hand, if the exact elastic equations were satisfied, a standard argument using the Bianchi identities would imply that also the harmonicity condition is satisfied and we have in fact solved the full field equations. On the other hand, if we could show that the full Einstein equations hold, the Bianchi identities would imply we also have a solution to the exact elastic equations.

In fact, none of these two alternatives are applicable, and one must prove both properties simultaneously. To do this a type of bootstrap argument must be used. It is worth mentioning that the boundary condition of vanishing normal traction is essential. We could in principle solve the projected elasticity equation together with the reduced field equations for a boundary condition which prescribes the position of the boundary in space. However, it would then in general not be possible to show that all the Einstein field equations are satisfied. This is consistent with the fact that fixing the surface of a body in space is not a physical problem in Einstein's theory.

The result of this paper is proved in a way which is completely different from the analogous result in our Newtonian paper [5]. In an appendix (Appendix B) we add an outline of the proof of the Newtonian result, found and kindly communicated to us by an anonymous referee, which exactly follows the pattern of the present work.

\section{PRELIMINARIES}

The following index conventions will be used. Upper case latin indices $A, B, C, \ldots$ take values $1,2,3$, lower case latin indices $i, j, k, \ldots$ take values $1,2,3$, and greek indices $\alpha, \beta, \gamma, \ldots$ take values $0,1,2,3$.

We will make use of Sobolev spaces $W^{k, p}$ on domains and the trace spaces $B^{s, p}$, as well as weighted Sobolev spaces $W_{\delta}^{k, p}$. Unless there is room for confusion, the same notation will be used for spaces of tensors and vectors, as for spaces of scalar functions. The rest of this section collects some notions and facts from analysis which shall be needed.

2.1. Sobolev spaces on domains. The books [1, 10] are general references for the material discussed in this section. For an integer $k \geq 0,1<p<\infty$ and a domain $\Omega, W^{k, p}(\Omega)$ is the closure of $C^{\infty}(\Omega)$ in the norm

$$
\|u\|_{W^{k, p}(\Omega)}=\sum_{|\alpha| \leq k}\left\|\partial^{\alpha} f\right\|_{L^{p}(\Omega)}
$$

Further, we shall need the Nikol'skii-Besov spaces $B^{s, p}=B_{p}^{s, p}$, which are the trace spaces for the Sobolev spaces. These are Banach spaces with norm defined on $\mathbb{R}^{n}$ for 
$s>0,1<p<\infty$, by

$$
\|u\|_{B^{s, p}}=\|u\|_{L^{p}}+\left(\int_{\mathbb{R}^{n}}|h|^{-(n+s p)}\left\|\Delta_{h}^{\sigma} u\right\|_{L^{p}}^{p} d h\right)^{1 / p}
$$

where $\sigma$ is the smallest integer strictly greater than $s$, and $\Delta_{h}$ is the difference operator. There are versions of many of the facts stated in this section also for $p=1$, and $p=\infty$, see the references given above. Note $B^{k, p} \neq W^{k, p}$, except for the case $p=2$. Let $k \geq 1$. A well known fact is that for a domain $\Omega \subset \mathbb{R}^{n}$ with $C^{k}$ boundary, then for $1<p<\infty$, the trace $\operatorname{tr}_{\partial \Omega}$ has the property

$$
\operatorname{tr}_{\partial \Omega} W^{k, p}(\Omega)=B^{k-1 / p, p}(\partial \Omega)
$$

We shall make use of this fact in the case $k=1$. Further, under these conditions, there is a bounded linear extension operator $E: W^{k, p}(\Omega) \rightarrow W^{k, p}\left(\mathbb{R}^{n}\right) \cap C^{\infty}\left(\mathbb{R}^{n} \backslash \bar{\Omega}\right)$. Here $\bar{\Omega}=\Omega \cup \partial \Omega$ denotes the closure of $\Omega$. In fact, for the last mentioned result to hold, it is sufficient to assume that $\Omega$ has a Lipschitz regular boundary.

2.2. The boundary problem of linearized elasticity. The book [30] is a general reference for the material discussed in this section. Let $A_{i}{ }^{j}{ }_{k}{ }^{l}$ be a fourth order elasticity tensor on a domain $\Omega \subset \mathbb{R}^{n}$, i.e. $A$ has symmetries

$$
A_{i}{ }^{j}{ }^{l}=A_{j}{ }^{i}{ }^{l}=A_{i}{ }^{j}{ }^{k}=A_{k}{ }^{l}{ }^{j}
$$

Let

and defined the operator $L$ by

$$
\sigma(u)_{i}{ }^{j}=A_{i}{ }^{j}{ }^{l} \partial_{l} u^{k}
$$

$$
L u_{i}=\partial_{j} \sigma(u)_{i}{ }^{j}
$$

$L$ is strongly elliptic if

$$
A_{i}{ }^{j}{ }_{k}{ }^{l} \eta^{i} \xi_{j} \eta^{k} \xi_{l}>0, \quad \text { for all } \xi, \eta \in \mathbb{R}^{n} .
$$

For applications in elasticity it is natural to assume there is a positive constant $\lambda$ such that for all symmetric $n \times n$ matrices $\psi^{i}{ }_{j}$

$$
\lambda|\psi|^{2} \leq\left|A_{i}{ }^{j}{ }_{k}{ }^{l} \psi^{i}{ }_{j} \psi^{k}{ }_{l}\right| \leq \lambda^{-1}|\psi|^{2},
$$

see [30, Chapter III] or [26, S 4.3]. The pointwise stability condition (2.1) implies strong ellipticity. We will assume that (2.1) holds for the rest of this section.

The Neumann type problem

$$
L u_{i}=b_{i}, \quad \operatorname{tr}_{\partial \Omega} \sigma(u)_{i}{ }^{j} n_{j}=\tau_{i}
$$

is equivalent to the statement that

$$
A(\phi, u)=-\int_{\Omega} \phi^{i} b_{i}+\int_{\partial \Omega} \phi^{i} \tau_{i}
$$

for all $\phi \in C^{\infty}(\Omega)$, where $A(u, v)$ is the symmetric bilinear form

$$
A(u, v)=\int_{\Omega} \partial_{j} v^{i} A_{i}{ }^{j}{ }_{k}{ }^{l} \partial_{l} u^{k}
$$

Let $H^{s}(\Omega)=B^{s, 2}(\Omega)$. Then $A$ defines a bounded quadratic form on $H^{1}(\Omega)$. The radical $Z$ of $A$ is the space of $\xi \in H^{1}(\Omega)$ such that $A(\xi, u)=0$ for all $u \in H^{1}(\Omega)$. It follows from (2.1) and the symmetry properties of $A$, that $Z$ consists of all Euclidean Killing fields $\xi$, i.e. fields of the form

$$
\xi^{i}=a^{i}+b^{i}{ }_{j} x^{j}, \quad a^{i}, b^{i}{ }_{j} \text { constants, } b^{i}{ }_{j}=-b^{j}{ }_{i}
$$


Let $H^{1}(\Omega)_{e}$ be the $L^{2}$ orthogonal complement of the radical, i.e. the space of $u \in H^{1}(\Omega)$ such that

$$
\int_{\Omega} \xi_{i} u^{i}=0, \quad \forall \xi \in Z
$$

Under the above conditions, the quadratic form $A(u, v)$ is coercive on $H^{1}(\Omega)_{e}$. This follows from the pointwise stability condition (2.1) and Korn's inequality, see [30, p. 92]. Thus, by the Lax-Milgram theorem, we have that for any $\left(b_{i}, \tau_{i}\right) \in H^{-1}(\Omega) \times H^{-1 / 2}(\partial \Omega)$ satisfying

$$
\int_{\Omega} \xi^{i} b_{i}-\int_{\partial \Omega} \xi^{i} \tau_{i}=0, \quad \forall \xi \in Z,
$$

there is a unique $u \in H^{1}(\Omega)_{e}$, which is a weak solution to (2.2), and which satisfies the estimate

$$
\|u\|_{H^{1}} \leq C\left(\|b\|_{H^{-1}(\Omega)}+\|\tau\|_{H^{-1 / 2}(\partial \Omega)}\right)
$$

We will later refer to (2.6) as an equilibration condition. The physical meaning of the equilibration condition is that the total force and torque exerted by $(b, \tau)$ is zero. Now, assuming $\partial \Omega \in C^{k+2}, A_{i}{ }^{j}{ }_{k}{ }^{l} \in C^{k+1}, u \in W^{k+2, p}(\Omega)$, one has from [2] an estimate of the form

$$
\|u\|_{W^{k+2, p}(\Omega)} \leq C\left(\|L(u)\|_{W^{k, p}(\Omega)}+\left\|\operatorname{tr}_{\partial \Omega} \sigma(u) \cdot n\right\|_{B^{1-1 / p, p}(\partial \Omega)}+\|u\|_{L^{p}(\Omega)}\right)
$$

Let $W^{k, p}(\Omega)_{e}$ be the space of $u \in W^{k, p}(\Omega)$ such that the condition (2.5) holds. Then we have by the above that for $b, \tau \in W^{k, p}(\Omega) \times B^{k+1-1 / p, p}(\partial \Omega)$, satisfying (2.6), there is a unique $u \in W^{k+2, p}(\Omega)_{e}$ which solves (2.2). In particular, in view of the above stated estimates, we have that the linear mapping $W^{k+2, p}(\Omega) \rightarrow W^{k, p}(\Omega) \times B^{k+1-1 / p, p}(\partial \Omega)$ defined by

$$
u \mapsto\left(L u, \operatorname{tr}_{\partial \Omega} \sigma(u) \cdot n\right)
$$

is Fredholm. It follows from the discussion above that the cokernel of the operator defined by (2.7) is defined by (2.6), and that the kernel consists of Killing fields, of the form given in (2.4).

2.3. Weighted Sobolev spaces on $\mathbb{R}^{n}$. The material which we shall need can be found in [3]. Let $n>2, r=|x|$, and let $\sigma=\left(1+r^{2}\right)^{1 / 2}$. For $k \geq 0, k$ integer, $1 \leq p \leq \infty, \delta \in \mathbb{R}$, define function spaces $W_{\delta}^{k, p}$ as the closure of $C_{0}^{\infty}\left(\mathbb{R}^{n}\right)$ in the norms

$$
\|u\|_{W_{\delta}^{k, p}}^{p}=\sum_{|\alpha| \leq k}\left\|\sigma^{|\alpha|-\delta-n / p} \partial^{\alpha} u\right\|_{L^{p}}^{p}
$$

We use the notation $L_{\delta}^{p}$ for $W_{\delta}^{0, p}$. Decreasing $\delta$ means faster decay. The spaces $W_{s-n / p}^{k, p}$ are equivalent to the homogenous Sobolev space $\dot{W}^{k, p}$ without weight, in particular $L_{-n / p}^{p}=$ $L^{p}$. The dual space of $W_{\delta}^{s, p}$ is $W_{\delta^{\prime}}^{-s, p^{\prime}}$ with $1 / p+1 / p^{\prime}=1, \delta^{\prime}=-\delta-n$. Weighted Hölder spaces $C_{\delta}^{k, \alpha}$ can be defined analogously, see [3].

Weighted function spaces can be analyzed in terms of ordinary function spaces by the following standard construction. Let $\phi$ be a bump function with support in $|x| \leq 2$ and which equals 1 on $|x| \leq 1$. Define a cutoff function $\psi$ by $\psi(x)=\phi(x)-\phi(2 x)$. Then 
$\psi$ has support in the annulus $1 / 2 \leq|x| \leq 2$. For a function $u$, let $u_{0}=\phi u$, and let $u_{i}(x)=\psi(x) u\left(2^{i} x\right)$ be the dilatations of $u$. An equivalent norm for $W_{\delta}^{k, p}$ is given by

$$
\|\| u\left\|_{W_{\delta}^{k, p}}^{p}=\sum_{i=0}^{\infty} 2^{-i p \delta}\right\| u_{i} \|_{W^{k, p}}^{p}
$$

Using this formulation, inequalities on compact domains may be systematically generalized to weighted spaces. The following are some of the basic inequalities for the weighted spaces.

(1) Inclusion: If $p_{1} \leq p_{2}, \delta_{2}<\delta_{1}$, and $u \in L_{\delta_{2}}^{p_{2}}$, then

$$
\|u\|_{L_{\delta_{1}}^{p_{1}}} \leq C\|u\|_{L_{\delta_{2}}^{p_{2}}}
$$

(2) Sobolev I: If $n-k p>0$ and $p \leq q \leq n p /(n-k p)$, then if $u \in W_{\delta}^{k, p \text {, }}$

$$
\|u\|_{L_{\delta}^{q}} \leq C|| u \|_{W_{\delta}^{k, p}}
$$

(3) Sobolev II: If $n<k p$, then if $u \in W_{\delta}^{k, p}$,

$$
\|u\|_{L_{\delta}^{\infty}} \leq C|| u \|_{W_{\delta}^{k, p}}
$$

and in fact $|u(x)|=o\left(r^{\delta}\right)$ as $r \rightarrow \infty$.

(4) Product estimate: If $n<k p$, and $u \in W_{\delta_{1}}^{k, p}, v \in W_{\delta_{2}}^{k, p}$, then with $\delta=\delta_{1}+\delta_{2}$,

$$
\|u v\|_{W_{\delta}^{k, p}} \leq C\|u\|_{W_{\delta_{1}}^{k, p}}\|v\|_{W_{\delta_{2}}^{k, p}}
$$

Let $\Delta$ denote the Laplacian defined with respect to the Euclidean metric on $\mathbb{R}^{n}$. We recall some facts about its mapping properties in the setting of weighted Sobolev spaces. Let $E=\{j: j$ integer $, j \neq 3-n, \ldots,-1\}$. In particular, for $n=3, E$ consists of all integers. The elements of $E$ are called exceptional weights. A weight $\delta \in \mathbb{R} \backslash E$ is called nonexceptional. Given $\delta \in \mathbb{R}$, define $\mathrm{k}^{-}(\delta)$ to be the largest exceptional weight $<\delta$.

A basic fact is that $\Delta: W_{\delta}^{k, p} \rightarrow W_{\delta-2}^{k-2, p}$ for $k \geq 2,1<p<\infty$, is Fredholm if and only if $\delta$ is nonexceptional. In particular, for $\delta \in(2-n, 0), \Delta: W_{\delta}^{k, p} \rightarrow W_{\delta-2}^{k-2, p}$ is an isomorphism. Let the operator $L$ be of the form $L=a^{i j} \partial_{i} \partial_{j}+b^{i} \partial_{i}+c$. Then with $q>n$, we will say that $L$ is asymptotic to $\Delta$, of order $\tau<0$, if

$$
a^{i j}-\delta^{i j} \in W_{\tau}^{2, q}, \quad b^{i} \in W_{\tau-1}^{1, q}, \quad c \in L_{\tau-2}^{q}
$$

Note that the above conditions are stronger than those stated in [3, Definition 1.5], and that we use the opposite sign convention for $\tau$. If $L$ is asymptotic to $\Delta$, then for $1<p \leq q$, $L: W_{\delta}^{2, p} \rightarrow L_{\delta}^{p}$ is bounded.

The following version of elliptic regularity is easily proved using standard estimates for elliptic operators on domains (see for example [14, Chapters 8,9]) and scaling. Suppose $L$ is asymptotic to $\Delta$ of order $\tau<0$ and suppose $2 \leq p \leq q$. For $u \in W_{\delta}^{1, p}$, such that $L u \in L_{\delta-2}^{p}$, elliptic regularity gives $u \in W_{\delta}^{2, p}$ and the inequality

$$
\|u\|_{W_{\delta}^{2, p}} \leq C\left(\|L u\|_{L_{\delta-2}^{p}}+\|u\|_{L_{\delta}^{p}}\right)
$$

holds. See [3, Proposition 1.6] for a stronger version of elliptic regularity.

If $\delta$ is nonexceptional, there are constants $C, R$ so that if $u \in W_{\delta}^{1, p}$, the scale broken estimate

$$
\|u\|_{W_{\delta}^{2, p}} \leq C\left(\|L u\|_{L_{\delta-2}^{p}}+\|u\|_{L^{p}\left(B_{R}\right)}\right)
$$

holds, cf. [3, Theorem 1.10]. Here $B_{R}=\{x:|x| \leq R\}$. We will now state a consequence of this estimate which we shall make use of. Assume that $\delta+\tau$ is non-exceptional. If 
$u \in W_{\delta}^{2, p}, L u \in L_{\delta+\tau-2}^{p}$, then for exceptional values $\delta+\tau<j \leq \mathrm{k}^{-}(\delta)$, there are $h^{j} \in C^{\infty}\left(\mathbb{R}^{n}\right)$, harmonic and homogenous of order $j$ in $\mathbb{R}^{n} \backslash B_{R}$, such that

$$
u=\sum_{\delta+\tau<j \leq \mathrm{k}^{-}(\delta)} h^{j}+v
$$

where $v \in W_{\delta+\tau}^{2, p}$, and an estimate of the form

$$
\sum_{j}\left\|h^{j}\right\|_{j}+\|v\|_{W_{\delta+\tau}^{2, p}} \leq C\left(\|L u\|_{L_{\delta+\tau-2}^{p}}+\|v\|_{B_{R}}\right)
$$

holds. Here $\left\|h^{j}\right\|_{j}$ is a suitable norm of for homogenous, harmonic functions, for example $\left\|h^{j}\right\|_{j}=\left\|r^{-j} h^{j}\right\|_{L^{\infty}\left(S_{2 R}\right)}$, with $S_{2 R}=\{x:|x|=2 R\}$. In particular, if ker $L=0$ on $W_{\delta}^{2, p}$, then the above estimate takes the form

$$
\sum_{j}\left\|h^{j}\right\|_{j}+\|v\|_{W_{\delta+\tau}^{2, p}} \leq C\|L u\|_{L_{\delta+\tau-2}^{p}}
$$

To make the above explicit, suppose $n=3,-1<\delta<0$ and $-2<\delta+\tau<-1$. Then $\mathrm{k}^{-}(\delta)=-1$ and with $u \in W_{\delta}^{2, p}, L u \in L_{\delta+\tau-2}^{p}$, we have

$$
u=\frac{c_{1}}{r} \zeta+v
$$

where $\zeta$ is a cutoff function such that $\zeta=1$ in $\mathbb{R}^{3} \backslash B_{R}$, and $\zeta=0$ in $B_{R / 2}$, and where $v \in W_{\delta+\tau}^{2, p}$ satisfies an estimate of the form

$$
\left|c_{1}\right|+\|v\|_{W_{\delta+\tau}^{2, p}} \leq C\left(\|L u\|_{L_{\delta+\tau-2}^{p}}+\|v\|_{B_{R}}\right)
$$

We shall make use of this estimate in section 5.1 .

For $\tau<0, p>n$, define the space $E_{\tau}^{k, p}$ of asymptotically Euclidean metrics on $\mathbb{R}^{n}$ as the space of $h_{i j}$ such that

$$
h_{i j}-\delta_{i j} \in W_{\tau}^{k, p}
$$

where $\delta_{i j}$ denotes the flat Euclidean metric on $\mathbb{R}^{n}$. Then $E_{\tau}^{k, p}$ is a Banach manifold.

Let $R_{i j}$ be the Ricci tensor of $h_{i j}$. We shall make use of the fact that if $h \in E_{\tau}^{2, p}$ for $p>n$, the operators $\Delta_{h}$ and $V^{i} \mapsto L V_{i}=\Delta_{h} V_{i}+R_{i j} V^{j}$ are asymptotic to $\Delta$ of order $\tau$. Here it should be noted that the principal part of the operator $L$ is the scalar Laplacian, acting diagonally. The results concerning elliptic operators that we have stated in this subsection generalize immediately to the case of elliptic systems of diagonal form.

2.4. Bianchi identity. The Bianchi identity for weakly regular Riemann spaces will play an important role in this paper, and therefore we give a proof of this fact below. The considerations in this subsection are local, and we work in local Sobolev spaces, denoted by $W_{\mathrm{loc}}^{k, p}$. By definition, $f \in W_{\mathrm{loc}}^{k, p}$ if for each compact domain $U \subset M, f \in W^{k, p}(U)$. For $1<p<\infty$, let $q$ be the dual exponent of $p$, such that $1 / p+1 / q=1$. For non-negative integers $k$, we define $W_{\mathrm{loc}}^{-k, q}$ as the dual space to $W_{\mathrm{loc}}^{k, p}$. Then $\partial^{\alpha} f \in W_{\mathrm{loc}}^{-k, p}$ if $f \in L_{\mathrm{loc}}^{p}$, where $k=|\alpha|$.

We make note of the following product estimates. Suppose that $p>n$. If $u \in W_{\mathrm{loc}}^{2, p}, v \in$ $W_{\mathrm{loc}}^{1, q}$, then $u v \in W_{\mathrm{loc}}^{1, q}$. To see this, differentiate the product and use Sobolev imbedding. Further, if $u \in W_{\mathrm{loc}}^{2, p}, w \in W_{\mathrm{loc}}^{-1, p}$, then $u w \in W_{\mathrm{loc}}^{-1, p}$. We estimate the product $u w$ as follows. Let $v \in W_{\text {loc }}^{1, q}$ and consider for any domain $U$ with compact closure, $\int_{U} v u w$. By the above mentioned estimates, $v u \in W^{1, q}(U)$. Thus the integral is well defined, and the inequality $\left|\int_{U} v u w\right| \leq C\|v\|_{W^{1, q}(U)}\|u\|_{W^{2, p}(U)}\|w\|_{W^{-1, p}(U)}$ holds. But $v \in W^{1, q}(U)$ 
was arbitrary. It follows that $u w$ defines a bounded linear functional on $W^{1, q}(U)$, and hence $u w \in W_{\mathrm{loc}}^{-1, p}$.

Lemma 2.1 (Bianchi identities). Consider a Riemann manifold $\left(M, h_{i j}\right)$ of dimension $n$, with metric $h_{i j} \in W_{\mathrm{loc}}^{2, p}, p>n$. Then the first Bianchi identity holds for $R_{i j k l}$. Further, the second Bianchi identity

$$
\nabla_{[m} R_{i j] k l}=0
$$

and the contracted second Bianchi identity

$$
\nabla^{i} R_{i j}-\frac{1}{2} \nabla_{j} R=0
$$

are valid in the sense of distributions.

Proof. The first two statements are clear from the product estimates and the definition of $\nabla$ and $R_{i j k l}$. It is most convenient to prove the Bianchi identity using the Cartan formalism. Let $\theta$ be an orthornomal coframe. The structure equations are

$$
\begin{gathered}
d \theta+\omega \wedge \theta=0 \\
d \omega+\omega \wedge \omega=\Omega
\end{gathered}
$$

Assuming $h_{i j} \in W_{\mathrm{loc}}^{2, p}$ we have $\omega \in W_{\mathrm{loc}}^{1, p}$ and $\Omega \in L_{\mathrm{loc}}^{p}$.

The Bianchi identity is the statement $d^{\omega} d^{\omega} d^{\omega}=0$, where $d^{\omega}$ is the covariant exterior derivative. Recall that on a section of a tensor bundle, $d^{\omega} d^{\omega} s=\left(d \omega+\frac{1}{2}[\omega \wedge \omega]\right) s=\Omega s$ and on a $\mathfrak{s o}(n)$-valued tensor, such as $\Omega, d^{\omega} H=d H+[\omega \wedge H]$. Evaluating $d^{\omega} d^{\omega} d^{\omega}$ gives

$$
\begin{aligned}
d^{\omega} \Omega & =d \Omega+[\omega \wedge \Omega] \\
& =d^{2} \omega+\frac{1}{2} d[\omega \wedge \omega]+[\omega \wedge \Omega]
\end{aligned}
$$

Now, $d^{2}=0$ on distributions. Further, expanding the other terms in the right hand side gives products of elements of $W_{\mathrm{loc}}^{1, p}$ and $L_{\mathrm{loc}}^{p}$. Therefore the standard algebraic identities hold to show that $d^{\omega} \Omega=0$ in the sense of distributions. This is equivalent to the statement that $\nabla_{[m} R_{k l] i}^{j}=0$ in the sense of distributions. Contracting this identity twice gives by the standard argument (making use of the product estimates stated above, and the fact that $\nabla_{m} R_{k l i}{ }^{j} \in W_{\text {loc }}^{-1, p}$, to justify the contraction), the identity

$$
\nabla^{i} R_{i j}-\frac{1}{2} \nabla_{j} R=0
$$

which holds in the sense of distributions.

For a domain $\Omega$ with boundary $\partial \Omega$, let $\chi_{\Omega}$ denote the characteristic function of $\Omega$, and $\operatorname{tr}_{\partial \Omega}$ the trace to the boundary. The following Lemma characterizes the divergence free tensors supported on a domain.

Lemma 2.2. Let $\left(M, h_{i j}\right)$ be a Riemann manifold of dimension $n$ with metric of class $W_{\text {loc }}^{2, p}, p>n$. Let $\Omega$ be a bounded domain compactly contained in $M$. Assume that $\Omega$ has $C^{1}$ boundary $\partial \Omega$, and let $T_{i j}$ be a symmetric tensor of class $W_{\mathrm{loc}}^{1, p}$. Then

$$
\nabla^{i}\left(T_{i j} \chi_{\Omega}\right)=\left(\nabla^{i} T_{i j}\right) \chi_{\Omega} \in L^{p}
$$

if and only if the zero traction condition

$$
\left(\operatorname{tr}_{\partial \Omega} T_{i j}\right) n^{j}=0
$$


holds, where $n^{j}$ denotes the normal of $\partial \Omega$. In particular, the identity

$$
\nabla^{i}\left(T_{i j} \chi_{\Omega}\right)=0
$$

holds in the sense of distributions, if and only if $\left(\nabla^{i} T_{i j}\right) \chi_{\Omega}=0$ and the zero traction condition holds.

Proof. Let $Y^{i} \in C_{0}^{\infty}$. Then we have

$$
\begin{aligned}
\int_{M} Y^{j} \nabla^{i}\left(T_{i j} \chi_{\Omega}\right) & =-\int_{M} \nabla^{i} Y^{j} T_{i j} \\
& =-\int_{\Omega} \nabla^{i} Y^{j} T i j \\
& =\int_{\Omega} Y^{j} \nabla^{i} T_{i j}+\int_{\partial \Omega} Y^{j} T_{i j} n^{i}
\end{aligned}
$$

where $n^{i}$ is the outward normal of $\partial \Omega$. This implies that with $1 / p+1 / q=1$, the inequality

$$
\left|\int_{M} Y^{j} \nabla^{i}\left(T_{i j} \chi_{\Omega}\right)\right| \leq\|Y\|_{L^{q}}\left\|\left(\nabla^{i} T_{i j}\right) \chi_{\Omega}\right\|_{L^{p}}
$$

holds if and only if $\left(\operatorname{tr}_{\partial \Omega} T^{i j}\right) n^{j}=0$. This proves the Lemma.

\section{THE FIELD EQUATIONS FOR A STATIC, SELF-GRAVITATING ELASTIC BODY}

We first consider a variational formulation of a self-gravitating elastic body in a $3+1$ dimensional spacetime $\left(\mathcal{M}, g_{\alpha \beta}\right)$ and then specialize this to the static case. The body $\mathcal{B}$ is a 3-manifold, possibly with boundary. We use coordinates $X^{A}$ on $\mathcal{B}$, and $x^{\alpha}$ on spacetime. In the Eulerian formulation of elasticity, the body is described by configurations $f: \mathcal{M} \rightarrow$ $\mathcal{B}$. The total Lagrangian density for the Einstein-matter system under consideration is, setting the speed of light $c=1$ for convenience,

$$
\mathcal{L}=-\frac{1}{16 \pi G} \sqrt{-g} R_{g}+\sqrt{-g} \rho
$$

where $\rho=\rho(f, \partial f, g)$ is the energy density of the materical in its own rest frame. General covariance implies that $\rho$ is of the form $\rho=\rho\left(f^{A}, \gamma^{A B}\right)$ where $\gamma^{A B}=f^{A}{ }_{, \alpha} f^{B}{ }_{, \beta} g^{\alpha \beta}$, where $f_{, \alpha}^{A}=\partial_{\alpha} f^{A}$. Geometrically, this means the following: there is a function $\hat{\rho}$ on the bundle of contravariant, symmetric two-tensors over $\mathcal{B}$, and $\rho\left(f^{A}, \gamma^{A B}\right)$ is the composition $\hat{\rho} \circ f_{*}\left(g^{-1}\right)$, where $g^{-1}$ is the inverse metric and $f_{*}$ is pushforward under the map $f$, acting on contravariant two-tensors. For an equivalent description of elastic materials see [12, 13. These references require in addition that $\mathcal{B}$ be furnished with a crystalline structure, which is essentially a choice of three linearly independent vector fields on $\mathcal{B}$ describing (the continuum limit of) the crystal lattice. More precisely, a crystalline structure is a linear subspace of the space of vector fields on $\mathcal{B}$ with the following property: for all points of $\mathcal{B}$ the evaluation map, which sends vector fields on $\mathcal{B}$ to tangent vectors at this point of $\mathcal{B}$, is an isomorphism, when restricted to this subspace. Our assumptions, in section 3.3 below, will render such a choice of vector fields which Lie commute. This means there are no no dislocations in the crystal lattice.

We now specialize to the static case. Let $\mathcal{M}=\mathbb{R} \times M$, where the space manifold $M$ is diffeomorphic to Eucidean 3-space, $M \cong \mathbb{R}_{\mathcal{S}}^{3}$. Further, we take the body $\mathcal{B}$ to be a bounded open domain in Eucliden 3-space, $\mathcal{B} \subset \mathbb{R}_{\mathcal{B}}^{3}$. We refer to $\mathbb{R}_{\mathcal{B}}^{3}$ as the extended body, and will use coordinate $X^{A}$ also on $\mathbb{R}_{\mathcal{B}}^{3}$. The body $\mathcal{B}$ will be assumed to have smooth boundary 
$\partial \mathcal{B}$, and the closure $\mathcal{B} \cup \partial \mathcal{B}$ will be denoted by $\overline{\mathcal{B}}$. Letting $\left(x^{\alpha}\right)=\left(t, x^{i}\right)$ where $x^{i}$ are coordinates on $M$, we can write the static spacetime metric in the form

$$
g_{\alpha \beta} d x^{\alpha} d x^{\beta}=-e^{2 U} d t^{2}+e^{-2 U} h_{i j} d x^{i} d x^{j}
$$

where $U, h_{i j}$ depend only on $x^{i}$. Further, the configurations $f: M \rightarrow \mathcal{B}$ are assumed to depend only on $x^{i}$. Assuming that a volume form $V_{A B C}$, on $\mathcal{B}$ is given, we may introduce the particle number density $n$ by

$$
f^{A}{ }_{, i}(x) f^{B}{ }_{, j}(x) f^{C}{ }_{, k}(x) V_{A B C}(f(x))=n(x) \epsilon_{i j k}(x)
$$

where $\epsilon_{i j k}$ is the volume element of $h_{i j}$. Note that the actual number density defined with respect to the metric $e^{-2 U} h_{i j}$ is $e^{3 U} n$. We assume the configurations $f$ are orientation preserving in the sense that $n$ is positive on $f^{-1}(\mathcal{B})$.

Let

$$
H^{A B}=f^{A}{ }_{i} f^{B}{ }_{, j} h^{i j}
$$

so that $\gamma^{A B}=e^{2 U} H^{A B}$. Note that equation (3.2) implies

$$
6 n^{2}=H^{A A^{\prime}} H^{B B^{\prime}} H^{C C^{\prime}} V_{A B C} V_{A^{\prime} B^{\prime} C^{\prime}}
$$

The Lagrangian density $\mathcal{L}$ is in terms of these variables, modulo a total divergence,

$$
\mathcal{L}=-\frac{1}{16 \pi G} \sqrt{h}\left(R-2|\nabla U|^{2}\right)+e^{U} n \epsilon \sqrt{h}
$$

Here, $R$ is the scalar curvature of $h_{i j},|\nabla U|^{2}=h^{i j} \nabla_{i} U \nabla_{j} U$, and the relativistic stored energy function $\epsilon$, defined by $\rho=n \epsilon$, is of the form $\epsilon=\epsilon\left(f^{A}, e^{2 U} H^{A B}\right)$, where $\epsilon$ is a smooth function of its arguments. In particular, by the chain rule, we have $\partial \epsilon / \partial H^{A B}=$ $e^{2 U} \partial \epsilon / \partial \gamma^{A B}$.

Suppose that a non-relativistic stored energy function $w\left(f^{A}, K^{A B}\right)$ is given, for example one suggested by experiment, where $K^{A B}$ is the non-relativistic analogue of $H^{A B}$. A relativistic stored energy function $\epsilon$ corresponding to $w$ can be defined as a sum of the specific rest mass $\stackrel{\circ}{\epsilon}$ and the relativistic analogue $w\left(f^{A}, e^{2 U} H^{A B}\right)$ of the stored energy function. The specific rest mass is defined such that $\stackrel{\circ}{\epsilon} V_{A B C}$ is the rest mass distribution of the material in its natural state. It can be shown that if the dependence on the light speed $c$ taken properly into account, the field equations tend to those of the corresponding Newtonian model when we let $c \nearrow \infty$. See [4] for details.

3.1. Field equations in Eulerian form. In order to write the field equations, we introduce the stress tensor $\sigma$. We will need the form of the stress tensor on the body and in space. These are given by

$$
\sigma_{A B}=-2 \frac{\partial \epsilon}{\partial H^{A B}}, \quad \sigma_{i j}=n f^{A}{ }_{, i} f^{B}{ }_{, j} \sigma_{A B}, \quad \sigma_{i}{ }^{A}=f^{B}{ }_{, i} \sigma_{B C} H^{C A}
$$

We remark that our convention for the stress here is that used in standard nonrelativistic elasticity, as opposed to the usual one in general relativity. It is important to note that the elastic quantities such as $H^{A B}, \sigma_{i j}$, viewed as functions on space, are only defined on $f^{-1}(\mathcal{B})$. The Euler-Lagrange equations resulting from the Lagrangian (3.4) are

$$
\begin{aligned}
\nabla_{j}\left(e^{U} \sigma_{i}{ }^{j}\right) & =e^{U}\left(n \epsilon-\sigma_{l}{ }^{l}\right) \nabla_{i} U \quad \text { in } f^{-1}(\mathcal{B}),\left.\quad \sigma_{i}{ }^{j} n_{j}\right|_{f^{-1}(\partial \mathcal{B})}=0 \\
\Delta_{h} U & =4 \pi G e^{U}\left(n \epsilon-\sigma_{l}{ }^{l}\right) \chi_{f^{-1}(\mathcal{B})} \quad \text { in } \mathbb{R}_{\mathcal{S}}^{3} \\
G_{i j} & =8 \pi G\left(\Theta_{i j}-e^{U} \sigma_{i j} \chi_{f^{-1}(\mathcal{B})}\right) \quad \text { in } \mathbb{R}_{\mathcal{S}}^{3}
\end{aligned}
$$


where

$$
\Theta_{i j}=\frac{1}{4 \pi G}\left[\nabla_{i} U \nabla_{j} U-\frac{1}{2} h_{i j}|\nabla U|^{2}\right]
$$

The system 3.6 is equivalent to the 4-dimensional Einstein equations

$$
G_{\mu \nu}=8 \pi G T_{\mu \nu}
$$

where $G_{\mu \nu}$ is the Einstein tensor of the static Lorentz metric given by (3.1) on $\mathbb{R} \times M$, and

$$
T_{\mu \nu} d x^{\mu} d x^{\nu}=e^{U}\left[e^{2 U} n \epsilon\left(u_{\mu} d x^{\mu}\right)^{2}-\sigma_{i j} d x^{i} d x^{j}\right], \quad u^{\mu} \partial_{\mu}=e^{-U} \partial_{t}
$$

The equations (3.6b/3.6c together imply the elasticity equation (3.6a). The reason is that the contracted Bianchi identity for $G_{i j}, \nabla^{i} G_{i j}=0$, implies that the right hand side of (3.6c) has vanishing divergence, and in particular the divergence of the compactly supported term $e^{U} \sigma_{i j} \chi_{f^{-1}(\mathcal{B})}$ must be well defined. By Lemma2.2 this implies that the zero traction boundary condition in equation (3.6a) holds, and hence by equations (3.6b) and (3.7), equation (3.6a) follows.

Let $\hat{\delta}$ be a fixed background metric on $M$, which we will take to be Euclidean, and let $\hat{\Gamma}_{j k}^{i}$ be the Christoffel symbol of $\hat{\delta}$. Then, with

$$
V^{i}=h^{j k}\left(\Gamma_{j k}^{i}-\hat{\Gamma}_{j k}^{i}\right)
$$

$-V$ is the tension field of the identity map $\left(M, h_{i j}\right) \rightarrow(M, \hat{\delta})$, and we have the identity

$$
R_{i j}=-\frac{1}{2} \Delta_{h} h_{i j}+\nabla_{(i} V_{j)}+Q_{i j}(h, \partial h)
$$

where $\Delta_{h} h_{i j}$ is the scalar Laplacian defined with respect to $h_{i j}$, acting on the components of $h_{i j}$ and $Q_{i j}$ is quadratic in $\partial h$. We use the notation $t_{(i j)}=\frac{1}{2}\left(t_{i j}+t_{j i}\right)$ for the symmetrization of a tensor. In particular, $h_{i j} \mapsto R_{i j}-\nabla_{(i} V_{j)}$ is a quasilinear elliptic operator, while $h_{i j} \mapsto R_{i j}$ fails to be elliptic. This failure is essentially due to the covariance of $R_{i j}$. It follows that also the system (3.6) fails to be elliptic. In order to construct solutions to (3.6), we will replace equation (3.6c) by the reduced system which results from replacing $R_{i j}$ by $R_{i j}-\nabla_{(i} V_{j)}$. The modified system which we will consider is of the form

$$
-\frac{1}{2} \Delta_{h} h_{i j}+Q_{i j}(h, \partial h)=2 \nabla_{i} U \nabla_{j} U-8 \pi G e^{U}\left(\sigma_{i j}-h_{i j} \sigma_{l}^{l}\right) \chi_{f^{-1}(\mathcal{B})}
$$

3.2. Field equations in material form. In the Eulerian formulation above, the elasticity equation (3.6a) is a nonlinear system with Neumann type boundary conditions on the domain $f^{-1}(\mathcal{B})$ which depends on the unknown configuration $f$. We will avoid dealing directly with this "free boundary" aspect of the system (3.6) by passing to the material, or Lagrangian form of the system. In this picture the configurations $f: M \rightarrow \mathcal{B}$ are replaced by deformations, i.e maps $\phi: \mathcal{B} \rightarrow M$ satisfying $\phi=f^{-1}$ on $\mathcal{B}$. Recall that the body $\mathcal{B}$ is a bounded open domain in $\mathbb{R}_{\mathcal{B}}$, the extended body, and that $\mathcal{B}$ is assumed to have a smooth boundary $\partial \mathcal{B}$. We will assume throughout the rest of this paper that $\mathcal{B}$ is connected. See remark 4.4 for discussion on this point.

We assume given a diffeomorphism $\mathbf{i}: \mathbb{R}_{\mathcal{B}}^{3} \rightarrow \mathbb{R}_{\mathcal{S}}^{3}$, which is an isometry, $\mathbf{i}^{*} \hat{\delta}=\delta_{\mathcal{B}}$. In Cartesian coordinate systems $X^{A}$ on $\mathbb{R}_{\mathcal{B}}^{3}$ and $x^{i}$ on $\mathbb{R}_{\mathcal{S}}^{3}$ where $\delta_{\mathcal{B}}$ and $\hat{\delta}$ have components $\delta_{A B}$ and $\delta_{i j}$ respectively, $\mathbf{i}$ can be assumed to have the form $\mathbf{i}^{i}(X)=\delta_{A}^{i} X^{A}$, so that $\partial_{A} \mathbf{i}^{i}=\delta_{A}^{i}$. Since $\mathcal{B}$ has smooth boundary, functions on $\mathcal{B}$ can be extended to the whole space, and in particular, given $\phi$, there is an extension $\widehat{\phi}: \mathbb{R}_{\mathcal{B}}^{3} \rightarrow \mathbb{R}_{\mathcal{S}}^{3}$, depending smoothly on $\phi$, such that $\widehat{\phi}(X)=\mathbf{i}(X)$ for $X$ outside some large ball. 
In the material picture, the dependent variables $f, U, h_{i j}$ are replaced by the fields $\phi, \bar{U}, \overline{h_{i j}}$ which we now introduce. As mentioned above, $\phi$ is assumed to be a diffeomorphism $\mathcal{B} \rightarrow \phi(\mathcal{B}) \subset \mathbb{R}_{\mathcal{S}}^{3}$, and the extension $\widehat{\phi}$ of $\phi$, which depends real analytically on $\phi$, is used to define the fields $\bar{U}=U \circ \widehat{\phi}$, a function on $\mathbb{R}_{\mathcal{B}}^{3}$, and $\overline{h_{i j}}=h_{i j} \circ \widehat{\phi}$, a metric on $\mathbb{R}_{\mathcal{B}}^{3}$. We will use the same symbols for these fields restricted to $\mathcal{B}$.

Remark 3.1. It is important to note that $\overline{h_{i j}} \neq \widehat{\phi}^{*} h_{i j}$, since we are only pulling back the components of $h_{i j}$ in the coordinate system $\left(x^{i}\right)$, not the tensor itself. In particular, $\overline{h_{i j}}$ does not transform as a tensor and is more propertly viewed as a collection of scalars. See appendix $\mathrm{A}$ for discussion.

The equation (3.2) defining $n$ can be written in the form $f^{*} V=n \mu_{h}$. where $\mu_{h}$ is the volume element of $h$. Defining $J=n^{-1}$, we have $\phi^{*} \mu_{h}=J V$. The Piola transform of $\sigma_{i}{ }^{j}$ can now be written in the form

$$
\bar{\sigma}_{i}{ }^{A}=J\left(f^{A}{ }_{, j} \sigma_{i}{ }^{j}\right) \circ \phi
$$

With this notation we have in particular the relation $\nabla_{A} \bar{\sigma}_{i}{ }^{A}=J\left(\nabla_{j} \sigma_{i}{ }^{j}\right) \circ \phi$. To derive the material version of (3.6a) one may use this relation directly, or proceed by first pulling back the matter Lagrangian to $\mathcal{B}$ and then applying the variational principle, see [6]. One finds

$$
\nabla_{A}\left(e^{\bar{U}} \bar{\sigma}_{i}{ }^{A}\right)=e^{\bar{U}}\left[\bar{\epsilon}-\frac{\bar{\sigma}_{l}^{l}}{\bar{n}}\right] \overline{\partial_{i} U} \quad \text { in } \mathcal{B},\left.\quad \bar{\sigma}_{i}{ }^{A} n_{A}\right|_{\partial \mathcal{B}}=0
$$

Here $\nabla_{A}\left(e^{\bar{U}} \bar{\sigma}_{i}{ }^{A}\right)$ is defined in terms of the volume element $V$ and does not involve a choice of metric on $\mathcal{B}$. We have

$$
\nabla_{A}\left(e^{\bar{U}} \bar{\sigma}_{i}{ }^{A}\right)=\frac{1}{V} \partial_{A}\left(V e^{\bar{U}} \bar{\sigma}_{i}{ }^{A}\right)-e^{\bar{U}} \phi^{j}{ }_{A} \bar{\Gamma}_{i j}^{k} \bar{\sigma}_{k}{ }^{A}
$$

The bars in Eq. (3.13) correspond to the convention that $f^{A}{ }_{i}$ be replaced by $\psi^{A}{ }_{i}$ defined as a functional of $\phi$ by

$$
\psi^{A}{ }_{i}(X) \widehat{\phi}^{i}, B(X)=\delta^{A}{ }_{B}
$$

and $H^{A B}$ be changed into

$$
\bar{H}^{A B}=\psi^{A}{ }_{i} \psi^{B}{ }_{j} \overline{h^{i j}},
$$

thus $\bar{H}^{A B}$ is the inverse of $\widehat{\phi}^{*} h_{i j}$. Note $\psi^{A}{ }_{i}$ is defined on $\mathbb{R}_{\mathcal{B}}^{3}$. With $\bar{\epsilon}=\bar{\epsilon}\left(X, e^{2 \bar{U}} \bar{H}^{A B}\right)$ and $\bar{H}^{A B}$ understood in this sense, we have the identity

$$
\bar{\sigma}_{i}{ }^{A}=\frac{\partial \bar{\epsilon}}{\partial \phi^{i}, A} .
$$

In particular, $\bar{\sigma}_{i}{ }^{A}=\psi^{B}{ }_{i} \bar{\sigma}_{B C} \bar{H}^{C A}$, and hence (3.13) is a second order equation for $\phi$. For barred quantities the corresponding rule for bars of derivatives gives for $U$

$$
\overline{\partial_{i} U}=\psi_{i}^{A} \partial_{A} \bar{U}
$$

For equations (3.6b) and (3.12) we simply replace each term by its barred version, i.e.

$$
\overline{\Delta_{h} U}=4 \pi G e^{\bar{U}}\left(\bar{n} \bar{\epsilon}-\bar{\sigma}_{l}^{l}\right) \chi_{\mathcal{B}} \quad \text { in } \mathbb{R}_{\mathcal{B}}^{3}
$$

Further, the covariance of the Laplacian implies $\overline{\Delta_{h} U}=\left(\Delta_{h} U\right) \circ \widehat{\phi}=\Delta_{\widehat{\phi}^{*} h}(U \circ \widehat{\phi})$, so that in this expression, the pullback $\widehat{\phi}^{*} h$ appears. This tensor is given by the inverse of 
$\bar{H}^{A B}$. It follows that $\overline{\Delta_{h} U}$ involves second derivatives of $\widehat{\phi}$. An analogous remark applies to

$$
-\frac{1}{2} \overline{\Delta_{h} h_{i j}}+Q_{i j}(\bar{h}, \overline{\partial h})=2 \overline{\left(\nabla_{i} U\right)\left(\nabla_{j} U\right)}-8 \pi G e^{\bar{U}}\left(\bar{\sigma}_{i j}-\overline{h_{i j}} \bar{\sigma}_{l}^{l}\right) \chi_{\mathcal{B}} .
$$

3.3. Constitutive conditions. We shall need to assume that the elastic material is able to relax in Euclidean space (which in particular implies the absence of dislocations, see the remark at the beginning of section 3). Further, the stored energy function $\epsilon$ must be such that the linearized elasticity operator is elliptic. In fact, we shall assume the pointwise stability condition. The constitutive conditions for $\epsilon$ are thus formulated as follows. There should exist a Euclidean metric $\delta_{\mathcal{B}}=\delta_{A B} d X^{A} d X^{B}$ on $\mathcal{B}$ (we will use the same symbol for this metric extended to $\mathbb{R}_{\mathcal{B}}^{3}$ ) such that

$$
\stackrel{\circ}{\epsilon}(X)=\left.\epsilon\right|_{\left(U=0, H=\delta_{\mathcal{B}}\right)} \geq C,\left.\quad\left(\frac{\partial \epsilon}{\partial H^{A B}}\right)\right|_{\left(U=0, H=\delta_{\mathcal{B}}\right)}=0 \text { in } \mathcal{B}
$$

and

$$
\stackrel{\circ}{L}_{A B C D} N^{A B} N^{C D} \geq C^{\prime}\left(\delta_{C A} \delta_{B D}+\delta_{C B} \delta_{A D}\right) N^{A B} N^{C D} \text { in } \mathcal{B},
$$

where

$$
{\stackrel{\circ}{L_{A B C D}}}(X):=\left.\left(\frac{\partial^{2} \epsilon}{\partial H^{A B} \partial H^{C D}}\right)\right|_{\left(U=0, H=\delta_{\mathcal{B}}\right)} .
$$

and $C, C^{\prime}$ are positive constants. The condition $\underline{3.21}$ is just the pointwise stability condition (2.1) discussed in section 2.2. The quantity $\stackrel{\circ}{\epsilon}$ appearing in (3.20) is the rest mass term in the relativistic stored energy function, as discussed above. For physical reasons, and in fact for hyperbolicity of the time dependent theory, it is necessary to assume that $C$ is positive. However, for the purposes of the present paper, this condition could be dropped. We shall assume, for simplicity, that $V_{A B C}$ is the volume form associated with $\delta_{\mathcal{B}}$ (i.e. that $V_{123}=1$ in Euclidean coordinates), so that $n \sqrt{h}=\operatorname{det}(\partial f)$.

\section{AnAlytical SETTING}

We will use the implicit function theorem to construct, for small values of Newton's constant $G$, static self-gravitating elastic bodies near the reference state described in section 3.3. We will use the field equations in the material form given by (3.13), (3.18) and (3.19).

We will now introduce the analytical setting where this work will be carried out. Fix a weight $\delta \in(-1,1 / 2)$. This choice of $\delta$ determines the weighted Sobolev spaces which will be used in the implicit function theorem argument below. The range of weights for which the isomorphism property for $\Delta$ holds is $(-1,0)$ but we shall need $\delta \in(-1,1 / 2)$ later on. Further, we fix $p>3$ to be used in setting up the function spaces which will appear in our argument. The body $\mathcal{B}$ is a bounded open domain in $\mathbb{R}_{\mathcal{B}}$, the extended body, with smooth boundary $\partial \mathcal{B}$. Under these conditions, the trace to the boundary $\operatorname{tr}_{\partial \mathcal{B}}$ is a continuous linear map $W^{1, p}(\mathcal{B}) \rightarrow B^{1-1 / p, p}(\partial \mathcal{B})$, and there is a bounded, linear extension operator $E$ : $W^{2, p}(\mathcal{B}) \rightarrow W_{\text {loc }}^{2, p}\left(\mathbb{R}_{\mathcal{B}}^{3}\right)$, see the discussion in section 2.1 or [1]. The spaces which will be used in the implicit function theorem argument are $B_{1}=W^{2, p}(\mathcal{B}) \times W_{\delta}^{2, p} \times E_{\delta}^{2, p}$, and let $B_{2}=\left[L^{p}(\mathcal{B}) \times B^{1-1 / p, p}(\partial \mathcal{B})\right] \times L_{\delta-2}^{p} \times L_{\delta-2}^{p}$. Thus, $B_{1}$ is a Banach manifold, and $B_{2}$ is a Banach space.

The residuals of equations (3.13), (3.18) and (3.19) define a map $\mathcal{F}: \mathbb{R} \times B_{1} \rightarrow$ $B_{2}, \mathcal{F}=\mathcal{F}(G, Z)$, where we use $Z=\left(\phi, \bar{U}, \overline{h_{i j}}\right)$ to denote a general element of $B_{1}$. 
We assume that $\phi$ is a diffeomorphism onto its image. Thus, $\mathcal{F}$ has components $\mathcal{F}=$ $\left(\mathcal{F}_{\phi}, \mathcal{F}_{U}, \mathcal{F}_{h}\right)$, corresponding to the components of $B_{2}$, given by

$$
\begin{aligned}
\mathcal{F}_{\phi} & =\left(\nabla_{A}\left(e^{\bar{U}} \bar{\sigma}_{j}{ }^{A}\right)-e^{\bar{U}}\left[\bar{\epsilon}-\frac{\bar{\sigma}_{l}{ }^{l}}{\bar{n}}\right] \overline{\partial_{i} U}, \quad \operatorname{tr}_{\partial \mathcal{B}}\left(\bar{\sigma}_{i}{ }^{A}\right) n_{A}\right) \\
\mathcal{F}_{U} & =\overline{\Delta_{h} U}-4 \pi G e^{\bar{U}}\left(\bar{n} \bar{\epsilon}-\bar{\sigma}_{l}^{l}\right) \chi_{\mathcal{B}} \\
\mathcal{F}_{h} & =-\frac{1}{2} \overline{\Delta_{h} h_{i j}}+Q_{i j}(\bar{h}, \overline{\partial h})-2 \overline{\nabla_{i} U \nabla_{j} U}+8 \pi G e^{\bar{U}}\left(\bar{\sigma}_{i j}-\overline{h_{i j}} \bar{\sigma}_{l}{ }^{l}\right) \chi_{\mathcal{B}}
\end{aligned}
$$

Recall from the discussion in section 3.2 that the extension $\widehat{\phi}$ is needed in the definition of $\mathcal{F}$. The proof of the following Lemma is a straightforward construction involving the use of the extension operator $E$ and a cutoff function, and is left to the reader.

Lemma 4.1. Fix some $X_{0} \in \mathcal{B}$. There are constants $\mu>0, R$ such that for each $\phi: \mathcal{B} \rightarrow$ $\mathbb{R}_{\mathcal{S}}^{3},\|\phi-\mathbf{i}\|_{W_{\mathcal{B}}^{2, p}} \leq \mu$, there is an extension $\widehat{\phi}: \mathbb{R}_{\mathcal{B}}^{3} \rightarrow \mathbb{R}_{\mathcal{S}}^{3}$, which depends real analytically on $\phi$. The extension $\widehat{\phi}$ can be chosen such that the map $\phi \mapsto \widehat{\phi}$ is given by a continuous linear map from $W^{2, p}(\mathcal{B})$ to $W^{2, p}\left(B_{R}\left(X_{0}\right)\right), \widehat{\phi}(X)=\mathbf{i}(X)$ for $X \in \mathbb{R}_{\mathcal{B}}^{3} \backslash B_{R}\left(X_{0}\right)$, and $\widehat{\phi}^{-1} \in W_{\text {loc }}^{2, p}\left(\mathbb{R}_{\mathcal{S}}^{3}\right)$.

The equation to be solved is $\mathcal{F}(G, Z)=0$. The material form of the reference state is given by

$$
Z_{0}=\left(\mathbf{i}, 0, \hat{\delta}_{i j} \circ \mathbf{i}\right) \in B_{1} .
$$

The map $\mathcal{F}$ defined by (4.1) is easily verified to satisfy $\mathcal{F}\left(0, Z_{0}\right)=0$ and to map $B_{1} \rightarrow B_{2}$ locally near the reference state $Z_{0}$.

4.1. Differentiability of $\mathcal{F}$. In order to apply the implicit function theorem, we must verify that $\mathcal{F}$ is $C^{1}$ as a map $\mathbb{R} \times B_{1} \rightarrow B_{2}$, in the arguments $(G, Z), Z=\left(\phi, \bar{U}, \overline{h_{i j}}\right)$, near $\left(0, Z_{0}\right)$. In fact, $\mathcal{F}$ is real analytic if the stored energy function $\epsilon$ is real analytic) near $Z_{0}$. It is clear the dependence on $G$ is smooth. We will freely make use of the standard fact that if $f$ is a smooth function, and $u \in W^{1, p}(\mathcal{B}), p>3$, then $u \mapsto f(u)$ is a smooth mapping $W^{1, p}(\mathcal{B}) \rightarrow W^{1, p}(\mathcal{B})$, as well as the corresponding statement which holds for weighted Sobolev spaces. The map $u \mapsto f(u)$ is sometimes called a Nemytskii operator. We consider the dependence on $Z$ for each term separately.

It is straightforward to see that $\psi^{A}{ }_{i}$ depends smoothly on $\phi$. This means that in view of (3.16) and the smoothness of the stored energy function, for $\mathcal{F}_{\phi}$, we note that $\bar{\sigma}_{i}{ }^{A}$ depends smoothly on $Z$. Expanding the definition of $\mathcal{F}_{\phi}$ it is clear this depends smoothly on $Z$.

Next consider $\mathcal{F}_{U}$. Note that $\overline{\Delta_{h} U}$ is the covariant Laplacian in the metric $\bar{H}_{A B}$, where $\bar{H}_{A B}$ is the inverse of $\bar{H}^{A B}$ given by (3.15), and thus $\overline{\Delta_{h} U}$ depends smoothly on $Z$. Using the fact that $\bar{\sigma}_{i}{ }^{j}=\bar{n} \bar{\sigma}_{i}{ }^{A} \phi^{j}{ }_{, A}$, the lower order terms in $\mathcal{F}_{U}$ are seen to be smooth in $Z$

Finally, we consider $\mathcal{F}_{h}$. The discussion above of $\overline{\Delta_{h} U}$ applies also to $\overline{\Delta_{h} h_{i j}}$. The quadratic term $Q_{i j}(\bar{h}, \overline{\partial h})$ is evaluated by replacing each occurrence of $h_{i j}$ by $\overline{h_{i j}}$ and $\partial_{k} h_{i j}$ by

$$
\psi_{k}^{A} \partial_{A} \overline{h_{i j}}
$$

In view of the regularity assumptions we find that $Q_{i j}(\bar{h}, \overline{\partial h})$ is smooth in $Z$. It is straighforward to analyze the remaining terms in $\mathcal{F}_{h}$ in the same manner. We have now proved

Lemma 4.2. The map $\mathcal{F}: B_{1} \rightarrow B_{2}$ is $C^{1}$ near $\left(0, Z_{0}\right)$. 
4.2. The Frechet derivative $D_{2} \mathcal{F}\left(0, Z_{0}\right)$. Next we calculate the Frechet derivative $D_{2} \mathcal{F}\left(0, Z_{0}\right)$ and consider its properties. As we shall see, this derivative is not an isomorphism $T_{Z_{0}} B_{1} \rightarrow$ $B_{2}$, and therefore it will be necessary to modify the system of equations by applying a projection before applying the implicit function theorem.

We will denote by $\zeta, v, k$ the infinitesimal variations of the fields $\phi, \bar{U}, \overline{h_{i j}}$. Using the notation of section 3.3 , let

$$
\stackrel{\circ}{S}_{i j}^{A B}(X)=\delta^{A E} \delta^{B F} \delta^{C}{ }_{i} \delta^{D}{ }_{j} \stackrel{\circ}{L}_{C E D F}(X) .
$$

The tensor $\stackrel{\circ}{S}_{i j}^{A}{ }^{B}$ corresponds to the tensor $A_{i}{ }^{j}{ }_{k}{ }^{l}$ of section 2.2 A calculation shows

$$
\begin{aligned}
D_{\phi} \bar{\sigma}_{i}{ }^{A}\left(0, Z_{0}\right) \cdot \zeta & =\stackrel{\circ}{S}_{i}{ }_{j}{ }^{B} \partial_{B} \zeta^{j} \\
D_{\bar{U}} \bar{\sigma}_{i}{ }^{A}\left(0, Z_{0}\right) \cdot v & =2 \stackrel{S}{S}_{i}{ }^{A}{ }_{j}{ }^{B} \delta_{B}^{j} v \\
D_{\bar{h}} \bar{\sigma}_{i}{ }^{A}\left(0, Z_{0}\right) \cdot k & =\stackrel{\leftrightarrow}{S}_{i}{ }_{i}{ }_{j}{ }^{B} \delta^{l m} \delta_{B}^{n} k_{m n}
\end{aligned}
$$

Let $\xi^{i}$ be a Killing field in the reference metric $\hat{\delta}_{i j}$, in Euclidean coordinates $\xi^{i}(x)=$ $\alpha^{i}+\beta_{j}^{i} x^{j}$, with $\alpha^{i}, \beta_{j}^{i}$ constants, $\beta_{j}^{i}=-\beta_{i}^{j}$, and define $\xi^{i}(X)=\xi^{i}(\mathbf{i}(X))$. Then we have $\partial_{A} \xi^{i}=\beta_{k}^{i} \delta_{A}^{k}$, and hence due to the antisymmetry of $\beta^{i}{ }_{k}$,

$$
\partial_{A} \xi^{i} \stackrel{\circ}{S}_{i}^{A}{ }_{j}^{B} \equiv 0
$$

Let now $\delta \bar{\sigma}_{i}{ }^{A}$ denote any combination of the Frechet derivatives of $\bar{\sigma}_{i}{ }^{A}$, evaluated at $\left(0, Z_{0}\right)$. Assuming we use a coordinate system $X^{A}$ where $V_{123}=1$, we have due to (4.2), and Stokes' theorem, the important relation

$$
0=\int_{\mathcal{B}} \xi^{i} \partial_{A}\left(\delta \bar{\sigma}_{i}{ }^{A}\right)-\int_{\partial \mathcal{B}} \xi^{i}\left(\delta \bar{\sigma}_{i}{ }^{A}\right) n_{A},
$$

where $n^{A}$ is the outward normal. This can be interpreted as saying that due to the natural boundary conditions, the linearized elasticity operator is automatically equilibrated at the reference configuration $\left(0, Z_{0}\right)$. It follows from the constitutive conditions stated in section 3.3. that $\zeta \mapsto D_{\phi} \mathcal{F}_{\phi}\left(0, Z_{0}\right) \zeta$ is elliptic, cf. the discussion in section 2.2. Therefore, the operator

$$
D_{\phi} \mathcal{F}_{\phi}\left(0, Z_{0}\right): W^{2, p}(\mathcal{B}) \rightarrow\left[L^{p}(\mathcal{B}) \times B^{1-1 / p, p}(\partial \mathcal{B})\right]
$$

is Fredholm with kernel consisting of the Killing fields on $\mathcal{B}$, and cokernel (in the sense of the natural $L^{2}$ pairing) consisting of the Killing fields on $\mathbf{i}(\mathcal{B})$, as above.

The only nonzero contribution from the Frechet derivative of the first order term

$$
-e^{\bar{U}}\left[\bar{\epsilon}-\frac{\bar{\sigma}_{l}^{l}}{\bar{n}}\right] \overline{\partial_{i} U}
$$

in $\mathcal{F}_{\phi}$ is

$$
\left.D_{\bar{U}}\left(-e^{\bar{U}}\left[\bar{\epsilon}-\frac{\bar{\sigma}_{l}^{l}}{\bar{n}}\right] \overline{\partial_{i} U}\right)\right|_{\left(0, Z_{0}\right)} . v=-\stackrel{\epsilon}{\epsilon} \partial_{i} v
$$

This is a lower order term which cannot affect the Fredholm property of $D_{2} \mathcal{F}$, but it should be noted that it is not a priori equilibrated, and therefore in general does not take values in the range of $D_{\phi} \mathcal{F}_{\phi}$. By the above discussion we have

$$
D_{2} \mathcal{F}_{\phi}\left(0, Z_{0}\right)=\left(\partial_{A}\left(\delta \bar{\sigma}_{i}{ }^{A}\right)-\stackrel{\circ}{\epsilon} \partial_{i} v, \quad \operatorname{tr}_{\partial \mathcal{B}}\left(\delta \bar{\sigma}_{i}{ }^{A}\right) n_{A}\right)
$$

Considering the other components of $D_{2} \mathcal{F}$, the only nonzero terms are the diagonal entries $D_{\bar{U}} \mathcal{F}_{U}$ and $D_{\bar{h}} \mathcal{F}_{h}$. These are given by

$$
D_{\bar{U}} \mathcal{F}_{U} \cdot v=\Delta v
$$


and

$$
\left(D_{\bar{h}} \mathcal{F}_{h} \cdot k\right)_{i j}=-\frac{1}{2} \Delta k_{i j}
$$

where $\Delta=\delta^{A B} \partial_{A} \partial_{B}$ is the Laplacian in the Euclidean background metric on $\mathbb{R}_{\mathcal{B}}^{3}$. The operator $\Delta$ is an isomorphism $W_{\delta}^{2, p} \rightarrow L_{\delta-2}^{p}$ for $\delta \in(-1,0)$, cf. [3].

It follows from the above discussion that the Frechet derivative $D_{2} \mathcal{F}\left(0, Z_{0}\right)$ can be represented as the matrix of operators

$$
\left(\begin{array}{ccc}
D_{\phi} \mathcal{F}_{\phi} & D_{U} \mathcal{F}_{\phi} & D_{h} \mathcal{F}_{\phi} \\
0 & \Delta & 0 \\
0 & 0 & -\frac{1}{2} \Delta
\end{array}\right)
$$

(where the entries are evaluated at $\left(0, Z_{0}\right)$ ). In particular, the matrix is upper triangular, and the diagonal entries are isomorphisms, with the exception for $D_{\phi} \mathcal{F}_{\phi}\left(0, Z_{0}\right)$ which is Fredholm with nontrivial kernel and cokernel, cf. the discussion above. The off diagonal terms are bounded operators. Therefore, if we compose $\mathcal{F}$ with a projection which in the first component maps onto the range of $D_{\phi} \mathcal{F}_{\phi}\left(0, Z_{0}\right)$, and restrict the domain of definition of $\mathcal{F}$ to a subspace transverse to its kernel, the resulting map will have Frechet derivative at $\left(0, Z_{0}\right)$ which is an isomorphism, which will allow us to apply the implicit function theorem. The projection which will be used is introduced in the next section.

4.3. Projection. Introduce the projection operator $\mathbb{P}_{\mathcal{B}}: B_{2} \rightarrow B_{2}$, which acts as the identity in the second and third components of $B_{2}$ and is defined in the first component of $B_{2}$ as the unique projection along the cokernel of $D_{\phi} \mathcal{F}_{\phi}\left(0, Z_{0}\right)$ onto the range of $D_{\phi} \mathcal{F}_{\phi}\left(0, Z_{0}\right)$, which leaves the boundary data in the first component of $B_{2}$ unchanged. We use the the label $\mathcal{B}$ to indicate that $\mathbb{P}_{\mathcal{B}}$ operates on fields on the body and the extended body. We shall later need to transport the projection operator to fields on $\mathbb{R}_{\mathcal{S}}^{3}$.

In order to give the explicit form of the action of $\mathbb{P}_{\mathcal{B}}$ in the first component of $B_{2}$, let $\left(b_{i}, \tau_{i}\right)$ denote pairs of elements in $L^{p}(\mathcal{B}) \times B^{1-1 / p, p}(\partial \mathcal{B})$. By a slight abuse of notation, denote this operator too by $\mathbb{P}_{\mathcal{B}}$. We require that $\mathbb{P}_{\mathcal{B}}\left(b_{i}, \tau_{i}\right)=\left(b^{\prime}{ }_{i}, \tau_{i}\right)$, satisfying

$$
\int_{\mathcal{B}} \xi^{i} b^{\prime}{ }_{i}=\int_{\partial \mathcal{B}} \xi^{i} \tau_{i}
$$

for all Killing fields $\xi^{i}$. Pairs $\left(b^{\prime}{ }_{i}, \tau_{i}\right)$ satisfying this condition will be called equilibrated. Since $\mathbb{P}_{\mathcal{B}}$ is a projection along the cokernel, $b^{\prime}{ }_{i}$ must be of the form

$$
b_{i}^{\prime}=b_{i}-\eta_{i} \chi_{\mathcal{B}}
$$

where $\eta_{i}=\alpha_{i}+\beta_{i j} X^{j}$, with $\alpha_{i}, \beta_{i j}$ constants satisfying $\beta_{i j}=-\beta_{j i}$. We remark that the bilinear pairing $(\xi, \eta) \mapsto \int_{\mathcal{B}} \xi^{i} \eta_{i}$ is nondegenerate, it is simply the $L^{2}(\mathcal{B})$ inner product on the space of Killing fields. Further, the map

$$
\xi \mapsto \int_{\mathcal{B}} \xi^{i} b_{i}-\int_{\partial \mathcal{B}} \xi^{i} \tau_{i}
$$

defines a linear functional on the space of killing fields, for each pair $\left(b_{i}, \tau_{i}\right)$. Therefore, there is a unique $\eta_{i}$ of the form given above such that $b^{\prime}{ }_{i}=b_{i}-\eta_{i} \chi_{\mathcal{B}}$ satisfies (4.3) for all Killing fields $\xi^{i}$. 
4.4. Existence of solutions to the projected system. We are now in a position to apply the implicit function theorem to prove

Proposition 4.3. Let $\mathcal{F}: B_{1} \rightarrow B_{2}$ be map defined by (4.1) and let $\mathbb{P}_{\mathcal{B}}$ be defined as in section 4.3 Then, for sufficiently small values of Newton's constant $G$, there is a solution $Z=Z(G)$, where $Z=\left(\phi, \bar{U}, \overline{h_{i j}}\right)$, to the reduced, projected equation for self-gravitating elastostatics given by

$$
\mathbb{P}_{\mathcal{B}} \mathcal{F}(G, Z)=0 \text {. }
$$

In particular, for any $\epsilon>0$, there is a $G>0$, such that $Z(G)$ satisfies the inequality

$$
\|\phi-\mathbf{i}\|_{W^{2, p}(\mathcal{B})}+\left\|\overline{h_{i j}}-\delta_{i j}\right\|_{W_{\delta}^{2, p}}+\|\bar{U}\|_{W_{\delta}^{2, p}}<\epsilon .
$$

Proof. Let $\mathbb{Y}$ denote the range of $\mathbb{P}_{\mathcal{B}}$ and let $\mathbb{X}$ be the subspace of $T_{Z_{0}} B_{1}$, such that $(\phi-$ i) ${ }^{i}\left(X_{0}\right)=0$ and $\delta^{C}{ }_{i} \delta_{C[A} \partial_{B]}(\phi-\mathbf{i})^{i}\left(X_{0}\right)=0$ holds at some point $X_{0} \in \mathcal{B}$. We have already shown that $\mathcal{F}: \mathbb{R} \times B_{1} \rightarrow B_{2}$ is $C^{1}$ and it follows from the definition of $\mathbb{P}_{\mathcal{B}}$ that $\mathbb{P}_{\mathcal{B}} \mathcal{F}: \mathbb{R} \times \mathbb{X} \rightarrow \mathbb{Y}$ is $C^{1}$. Since $\mathcal{F}\left(0, Z_{0}\right)=0$, the Frechet derivative of $\mathbb{P}_{\mathcal{B}} \mathcal{F}(G, Z)$ with respect to $Z$, evaluated at $\left(0, Z_{0}\right)$ is $\mathbb{P}_{\mathcal{B}} D_{2} \mathcal{F}\left(0, Z_{0}\right)$ which we will denote by $A$. It is clear from the discussion above that $A$ is Fredholm with trivial cokernel. Therefore all that remains to be checked is that $\operatorname{ker} A$ is trivial. To see this, note that $\operatorname{ker} D_{\phi} \mathcal{F}_{\phi}$ consists of Killing fields, by the discussion in section 2.2. Prescribing the value and antisymmetrized derivative of a Killing field at one point determines the Killing field in all of $\mathcal{B}$. This implies that $A: \mathbb{X} \rightarrow \mathbb{Y}$ has trivial kernel, and it is therefore an isomorphism. Thus, the implicit function theorem for Banach spaces [20] applies to prove the existence of solutions to the equation $\mathbb{P}_{\mathcal{B}} \mathcal{F}(G, Z)=0$, for small values of $G$. Since $Z(G)$ depends continuously on $G$ in $B_{2}$, the inequality (4.5) follows.

Remark 4.4. Up to this stage, the connectedness of the body $\mathcal{B}$ has not played at role in our arguments. In particular, in the case of a body with $N>1$ connected components, there is a collection of $N$ elastic fields $\phi$ and an extension common to all of them. The equilibration argument of section 5 below fails, however, for the simple reason that we have one equation, namely (5.15), but $N$ elastic fields.

In the next section, we study the solutions provided by Proposition 4.3 and prove that they in fact represent solutions of the full system (3.6).

\section{EQUiLIBRATION}

Let $\left(\phi, \bar{U}, \overline{h_{i j}}\right) \in B_{1}$ be a solution to the reduced, projected system (4.4) as in Proposition 4.3, and let $\widehat{\phi}$ be the extension of $\phi$ provided by Lemma 4.1. Define $U, h_{i j}$ by $U=\bar{U} \circ \widehat{\phi}^{-1}$ and $h_{i j}=\overline{h_{i j}} \circ \widehat{\phi}^{-1}$. Then $U$ and $h_{i j}$ are elements of $W_{\delta}^{2, p}\left(\mathbb{R}_{\mathcal{S}}^{3}\right)$ and $E_{\delta}^{2, p}\left(\mathbb{R}_{\mathcal{S}}^{3}\right)$, respectively. Further, we set $f=\widehat{\phi}^{-1}$. Then $f \in W_{\text {loc }}^{2, p}$ and $f=\mathbf{i}^{-1}$ outside a large ball.

Let $\widehat{\phi}$ be the extension of $\phi$ provided by Lemma 4.1, and let $U, h_{i j}$ be defined by $U=$ $\bar{U} \circ \widehat{\phi}^{-1}, h_{i j}=\overline{h_{i j}} \circ \widehat{\phi}^{-1}$. The following corollary to Proposition 4.3 is immediate in view of the composition properties of Sobolev functions.

Corollary 5.1. For any $\epsilon>0$, there is a $G>0$ such that the inequality

$$
\|\phi-\mathbf{i}\|_{W^{2, p}(\mathcal{B})}+\left\|h_{i j}-\delta_{i j}\right\|_{W_{\delta}^{2, p}}+\|U\|_{W_{\delta}^{2, p}}<\epsilon .
$$

holds. 
5.1. Eulerian form of the projected system. Next we introduce the projection operator in space which corresponds to $\mathbb{P}_{\mathcal{B}}$. From this point on, we are only interested in the action of $\mathbb{P}_{\mathcal{B}}$ in the first component of $B_{1}$. We will be dealing with solutions to the projected system $\mathbb{P}_{\mathcal{B}} \mathcal{F}=0$, in particular this means that the boundary condition $\left.\bar{\sigma}_{i}{ }^{A} n_{A}\right|_{\partial \mathcal{B}}=0$ will be satisfied, due to the nature of the projection operator defined in section 4.3. Therefore it is convenient to write $\mathbb{P}_{\mathcal{B}} b=\mathbb{P}_{\mathcal{B}}(b, 0)$.

Recall the change of variables formula

$$
\int_{\mathcal{B}} u(X) d V(X)=\int_{f^{-1}(\mathcal{B})} u \circ f(x) n(x) d \mu_{h}(x)
$$

which results from the definition of $n$ in equation (3.2). This implies

$$
\int_{\mathcal{B}} \xi^{i}(X) b_{i}(X) d V(X)=\int_{f^{-1}(\mathcal{B})} \xi^{i} \circ f(x) n(x) b_{i} \circ f(x) d \mu_{h}
$$

Letting $b_{i}=\nabla_{A}\left(e^{\bar{U}} \bar{\sigma}_{j}{ }^{A}\right)-e^{\bar{U}}\left(\bar{\epsilon}-\frac{\bar{\sigma}_{l}{ }^{l}}{\bar{n}}\right) \overline{\partial_{j} U}$, we have, using the properties of the Piola transform,

$$
n b_{i} \circ f=\nabla_{j}\left(e^{U} \sigma_{i}{ }^{j}\right)-e^{U}\left(n \epsilon-\sigma_{l}{ }^{l}\right) \partial_{i} U
$$

Based on this, we define the space version $\mathbb{P}_{f^{-1}(\mathcal{B})}$ of the projection $\mathbb{P}_{\mathcal{B}}$ by

$$
\mathbb{P}_{f^{-1}(\mathcal{B})}(n \cdot(b \circ f))=n\left(\mathbb{P}_{\mathcal{B}} b\right) \circ f
$$

$\mathbb{P}_{\mathcal{B}}$ is a projection by construction, and it follows from the definition of $\mathbb{P}_{f^{-1}(\mathcal{B})}$ that it also is a projection. Due to this definition, the equation $\mathbb{P}_{\mathcal{B}} \mathcal{F}_{\phi}=0$ implies $\mathbb{P}_{f^{-1}(\mathcal{B})}\left(n \mathcal{F}_{\phi} \circ f\right)=$ 0 . This relation is written more explicitely as equation (5.2a) below. Summarizing, the triple $\left(\phi, U, h_{i j}\right)$ we have constructed satisfies the following set of equations,

$$
\mathbb{P}_{f^{-1}(\mathcal{B})}\left(\nabla_{j}\left(e^{U} \sigma_{i}{ }^{j}\right)-e^{U}\left(n \epsilon-\sigma_{l}{ }^{l}\right) \partial_{i} U\right)=0 \quad \text { in } f^{-1}(\mathcal{B}),
$$

$$
\begin{aligned}
& \left.\sigma_{i}^{j} n_{j}\right|_{\partial f^{-1}(\mathcal{B})}=0 \quad \text { in } \partial f^{-1}(\mathcal{B}) \\
& \Delta_{h} U=4 \pi G e^{U}\left(n \epsilon-\sigma_{l}^{l}\right) \chi_{f^{-1}(\mathcal{B})} \quad \text { in } \mathbb{R}_{\mathcal{S}}^{3} \\
& G_{i j}-\left(\nabla_{(i} V_{j)}-\frac{1}{2} h_{i j} \nabla_{l} V^{l}\right)=8 \pi G\left(\Theta_{i j}-e^{U} \sigma_{i j} \chi_{f^{-1}(\mathcal{B})}\right) \text { in } \mathbb{R}_{\mathcal{S}}^{3}
\end{aligned}
$$

Recall that equation (5.2d) is equivalent to the space version of equation (3.19),

$$
-\frac{1}{2} \Delta_{h} h_{i j}+Q_{i j}(h, \partial h)=2 \nabla_{i} U \nabla_{j} U-8 \pi G e^{U}\left(\sigma_{i j}-h_{i j} \sigma_{l}{ }^{l}\right) \chi_{f^{-1}(\mathcal{B})} .
$$

Since the body is bounded, the right hand sides of equation $(5.2 \mathrm{c}$ ) and the contribution from the stress in equation (5.3) have compact support, and hence $U, h_{i j}$ satisfy the reduced vacuum static Einstein equations near spatial infinity. This implies that we can get more information about their asymptotic behavior than is a priori given from the implicit function theorem argument.

Lemma 5.2. $U, h_{i j}$ are of the form

$$
\begin{aligned}
U & =\frac{m_{U}}{r}+U_{(2)} \\
h_{i j} & =\delta_{i j}+\frac{\gamma_{i j}}{r}+h_{(2) i j}
\end{aligned}
$$


for constants $m_{U}, \gamma_{i j}$, with $h_{(2) i j}, U_{(2)} \in W_{2 \delta}^{2, p}$. Further, for sufficiently small $G$, we have the estimates

$$
\begin{array}{r}
\left\|U_{(2)}\right\|_{W_{2 \delta}^{2, p}}+\left|m_{U}\right| \leq C\left(\left\|h_{i j}-\delta_{i j}\right\|_{W_{\delta}^{2, p}}+\|\phi-\mathbf{i}\|_{W^{2, p}(\mathcal{B})}\right) \\
\left\|h_{(2) i j}\right\|_{W_{2 \delta}^{2, p}}+\|\gamma\| \leq C\left(\left\|h_{i j}-\delta_{i j}\right\|_{W_{\delta}^{2, p}}+\|\phi-\mathbf{i}\|_{W^{2, p}(\mathcal{B})}\right)
\end{array}
$$

where $\|\gamma\|=\left(\sum_{i, j} \gamma_{i j}^{2}\right)^{1 / 2}$.

Remark 5.3. If $h_{i j}, U$ were solutions of the full static vacuum Einstein equations near infinity, with the same falloff conditions, one could conclude that the $O(1 / r)$ term in $h_{i j}$ vanishes, following the argument in [17], see also [7]. However, in our situation $U, h_{i j}$ satisfies the reduced static vacuum Einstein equations near infinity and this argument does not apply directly.

Proof. By construction, we have $h_{i j}-\delta_{i j} \in W_{\delta}^{2, p}$ and $U \in W_{\delta}^{2, p}$, with $\delta \in(-1,-1 / 2)$. This, together with equation (5.2c) implies using the product estimates (2.9) to expand $\Delta_{h} U$, that

with

$$
\Delta_{e} U=f \in L_{2 \delta-2}^{p}
$$

$$
\begin{aligned}
\|f\|_{L_{2 \delta-2}^{p}} & \leq C\left(\left\|h_{i j}-\delta_{i j}\right\|_{W_{\delta}^{2, p}}+\left\|n \epsilon-\sigma_{l}^{l}\right\|_{L^{p}\left(f^{-1}(\mathcal{B})\right.}\right) \\
& \leq C\left(\left\|h_{i j}-\delta_{i j}\right\|_{W_{\delta}^{2, p}}+\|\phi-\mathbf{i}\|_{W^{2, p}(\mathcal{B})}\right)
\end{aligned}
$$

where in the last step we estimated $n \epsilon-\sigma_{l}{ }^{l}$ in terms of $h_{i j}, \phi-\mathbf{i}$.

We follow the proof of [3, Theorem 17], see also the discussion in section 2.3 Let $u$ satisfy

$$
\Delta_{e} u=f \in L_{2 \delta-2}^{p}
$$

By the isomorphism property of $\Delta_{e}: W_{\delta}^{2, p} \rightarrow L_{\delta-2}^{p}$, and the inclusion $L_{2 \delta-2}^{p} \subset L_{\delta-2}^{p}$, we have

$$
\|u\|_{W_{\delta}^{2, p}} \leq C\|f\|_{L_{2 \delta-2}^{p}}
$$

Fix some large radius $R$, and a point $x_{0} \in \mathbb{R}^{3}$. Let $B_{R}=B_{R}\left(x_{0}\right)$, and let $E_{R}=\mathbb{R}^{3}-$ $B_{R}$. By assumption, $\delta \in(-1,-1 / 2)$, so that $-2<2 \delta<-1$. In particular $2 \delta$ is nonexceptional, and hence $\Delta_{e}: W_{2 \delta}^{2, p} \rightarrow L_{2 \delta-2}^{p}$ is Fredholm. Hence there is a $v \in W_{2 \delta}^{2, p}$ such that

$$
\Delta_{e}(u-v)=0 \quad \text { in } E_{R}
$$

It follows that $u-v$ satisfies

$$
u-v=\frac{m}{r}+O\left(1 / r^{2}\right) \quad \text { in } E_{R}
$$

for some constant $m$. Here the term $O\left(1 / r^{2}\right)$ comes from the expansion of harmonic functions, and is in $W_{2 \delta}^{2, p}$. Let $\zeta$ be a cutoff function such that $\zeta=1$ in $E_{2 R}, \zeta=0$ in $B_{R}\left(x_{0}\right)$ and with $|\partial \zeta| \leq C / R$. Set

$$
w=u-\frac{m}{r} \zeta \in W_{2 \delta}^{2, p}
$$

Then we have $w=u$ in $B_{R}\left(x_{0}\right)$. Further, we have

$$
\Delta_{e} w=f-\Delta_{e}\left(\frac{m}{r} \zeta\right)
$$

where $\Delta_{e}\left(\frac{m}{r} \zeta\right)$ has compact support and we can estimate

$$
\|\left.\Delta_{e}\left(\frac{m}{r} \zeta\right)\right|_{L_{2 \delta-2}^{2}} \leq C|m|
$$


Since

$$
u=\Gamma \star f
$$

where $\Gamma=1 / r$ is the fundamental solution of $\Delta_{e}$ we have

$$
|m| \leq C|| f\left\|_{L^{1}\left(\mathbb{R}^{3}\right)} \leq C|| f\right\|_{L_{2 \delta-2}^{p}}
$$

Here we used the inclusion $L_{2 \delta-2}^{p} \subset L_{2 \delta-2}^{1} \subset L_{-3}^{1}$, and the fact that $L^{1}=L_{-3}^{1}$ in 3 dimensions. Thus we have

$$
\left\|\Delta_{e} w\right\|_{L_{2 \delta-2}^{p}} \leq C\|f\|_{L_{2 \delta-2}^{p}}
$$

Using the fact that ker $\Delta_{e}$ is trivial in $W_{2 \delta}^{2, p}$ together with the Fredholm property, we now have

$$
\|w\|_{W_{2 \delta}^{2, p}} \leq C\|f\|_{L_{2 \delta-2}^{p}}
$$

This argument proves (5.4) and (5.6). Applying the same argument to $h_{i j}$ shows

$$
h_{i j}=\delta_{i j}+\frac{\gamma_{i j}}{r}+h_{(2) i j}
$$

with $\gamma_{i j}$ constant and $h_{(2) i j} \in W_{2 \delta}^{2, p}$, and gives an estimate for $h_{(2) i j}, \gamma_{i j}$ in terms of $h_{i j}-\delta_{i j}, U$, and $\phi-\mathbf{i}$. This gives (5.7), since we have an estimate for $U$ in terms of $h_{i j}-\delta_{i j}, \phi-\mathbf{i}$ from (5.6).

5.2. The tension field. The next three lemmas state the properties of the tension field $V^{k}$, defined by (3.10), which we shall need. We may assume $\hat{\Gamma}_{j k}^{i}=0$. Let the operator $L$ be defined by

$$
L V^{k}=\Delta_{h} V^{k}+R_{j}^{k} V^{j}
$$

where $\Delta_{h}, R_{i j}$ are the covariant Laplacian and the Ricci tensor of $h_{i j}$, respectively.

Lemma 5.4. Assume we are in a Cartesian coordinate system so that $\hat{\delta}_{i j}=\delta_{i j}$ and $\hat{\Gamma}_{i j}^{k}=$ 0 . Let $r=|x|$ and set $\theta^{i}=x^{i} / r$. Let $h_{i j}$, $\gamma_{i j}$ be as in (5.5). Suppose $L V \in L_{\delta-3}^{p}$, where $V$ is the tension field of $h$. Then if $G$ is sufficiently small, $V \in W_{\delta-1}^{2, p}$ and the estimate

$$
\|V\|_{W_{\delta-1}^{2, p}} \leq C|| L V \|_{L_{\delta-3}^{p}}
$$

holds. Further, $V$ is of the form

$$
V^{k}=-\frac{1}{2 r^{2}}\left(\gamma_{j}^{k} \theta^{j}-\gamma_{m}^{m} \theta^{k}\right)+V_{(3)}^{k}
$$

where $V_{(3)}^{k} \in W_{2 \delta-1}^{2, p}$, and $\gamma^{i}{ }_{j}=\delta^{i k} \gamma_{k j}$.

Proof. The operator $L$ is an elliptic system, which is of diagonal type. Therefore the results discussed in section 2.3 apply to $L$. In particular, $L$ is asymptotic to $\Delta$ with rate $\delta$. Thus, $L: W_{\delta-1}^{2, p} \rightarrow L_{\delta-3}^{p}$ is Fredholm. It follows from the definition of $V$ and the assumptions on $h$ that $V \in W_{\delta-1}^{1, p}$. If $L V \in L_{\delta-3}^{p}$, then elliptic regularity gives $V \in W_{\delta-1}^{2, p}$. For small data $L$ has trivial kernel in this range of spaces, since $L$ is then a small perturbation of $\Delta_{e}$ which has trivial kernel in $W_{\tau}^{2, p}, \tau<0$. By Corollary 5.1 , for $G$ sufficiently small, the required condition on $L$ will hold. Therefore, if $G$ is sufficiently small, an estimate of the form 5.8 holds.

Recall from Lemma 5.2 that

$$
h_{i j}=\delta_{i j}+\frac{\gamma_{i j}}{r}+h_{(2) i j}
$$


with $h_{(2) i j} \in W_{2 \delta}^{2, p}$. Let

$$
H^{k}=-\frac{1}{2 r^{2}}\left(\gamma^{k}{ }_{j} \theta^{j}-\gamma^{m}{ }_{m} \theta^{k}\right) .
$$

Under our assumptions, $\hat{\Gamma}_{i j}^{k}=0$. From the definition of $V^{k}$, a calculation shows

$$
V^{k}-H^{k} \in W_{2 \delta-1}^{1, p},
$$

in particular $V^{k}-H^{k}=o\left(1 / r^{2 \delta-1}\right)$. The operator $L$ is asymptotic to $\Delta_{e}$ with rate $\delta$, see section 2.3 for this notion. It follows by an argument along the lines of the proof of Lemma 5.2 that

$$
V^{k}=H^{k}+V_{(3)}^{k}
$$

with $V_{(3)}^{k} \in W_{2 \delta-1}^{2, p}$. This completes the proof of the Lemma.

Let $\xi$ be a Euclidean Killing field of the form $\xi^{i}(X)=\xi^{i}(\mathbf{i}(X))$ with $\xi^{i}(x)=\alpha^{i}+$ $\beta^{i}{ }_{j} x^{j}$ for $\alpha^{i}, \beta^{i}{ }_{j}$ constants such that $\beta^{i}{ }_{j}=-\beta^{j}{ }_{i}$.

Lemma 5.5. Let $V$ be as in (5.9). Then the identity

$$
\int \xi^{i} \circ f L V_{i} d \mu_{h}=\int L\left(\xi^{i} \circ f\right) V_{i} d \mu_{h}
$$

holds.

Proof. First note that both sides of equation (5.11) are well defined. The integrand on the left hand side has compact support by equation (5.15). Recall that $f$ equals the identity map outside of a compact set. We have

$$
L(\xi \circ f) \in L_{\delta-1}^{p}
$$

and

$$
V_{i}=O\left(1 / r^{2}\right)
$$

Therefore the integrand in the right hand side is an element of $L_{\delta-3}^{p} \subset L^{1}$. In order to justify the partial integration, let $B_{R}=\{|x| \leq R\}$ and $S_{R}=\{|x|=R\}$. Then (5.11) is equivalent to

$$
\lim _{R \rightarrow \infty} \int_{B_{R}} \xi^{i} \circ f L V_{i} d \mu_{h}=\lim _{R \rightarrow \infty} \int_{B_{R}} L\left(\xi^{i} \circ f\right) V_{i} d \mu_{h} .
$$

Gauss law applied to the integral over $B_{R}$ gives

$$
\begin{aligned}
\int_{B_{R}} \xi^{i} \circ f L V_{i} d \mu_{h}= & \int_{B_{R}} L\left(\xi^{i} \circ f\right) V_{i} d \mu_{h} \\
& +\int_{S_{R}} \xi^{i} \circ f \nabla_{k} V^{i} n^{k} d A_{h}-\int_{S_{R}} \nabla_{k}\left(\xi^{i} \circ f\right) n^{k} V_{i} d A_{h}
\end{aligned}
$$

Let $\theta^{k}=x^{k} / r$. With the asymptotic behavior of $h_{i j}$ from Lemma 5.2 we have $n^{k}=$ $\theta^{k}+O(1 / r)$ and $\Gamma_{j k}^{i}=O\left(1 / r^{2}\right)$. Further, the area element induced on $S_{R}$ from $h_{i j}$ differs from the standard area element of $S_{R}$ by a term of order $O(1 / R)$. Recall that by Lemma 5.4 $V^{k}=H^{k}+V_{(3)}^{k}$ with $H^{k}$ given by (5.10) and $V_{(3)}^{k} \in W_{2 \delta-1}^{2, p}$. Since we are interested in the integral over $S_{R}$ in the limit $R \rightarrow \infty$, this shows that the only important terms in the boundary integrals in (5.12) are

$$
\int_{S_{R}} \beta^{k}{ }_{i} x^{i} \partial_{l} H_{k} \theta^{l} d A_{R}-\int_{S_{R}} \beta^{i}{ }_{k} H^{i} \theta^{k} d A_{R}
$$


We consider the first of these terms. Thus let $Z=\beta^{k}{ }_{i} x^{i} \partial_{l} H_{k} \theta^{l}$, the integrand in the first boundary integral above. At this point, we will be doing all calculations in the background metric $\delta_{i j}$, and therefore it is convenient to lower all indices using $\delta_{i j}$ and sum over repeated indices. A calculation shows

$$
\partial_{l} H_{k}=-\frac{1}{2 r^{3}}\left[2 \gamma_{l k}-\gamma_{m m} \delta_{l k}-3\left(2 \gamma_{j k} \theta_{l} \theta_{j}-\gamma_{m m} \theta_{l} \theta_{k}\right)\right]
$$

This gives using $\theta_{k} \theta_{k}=1$,

$$
Z=\beta_{k i} x_{i} \partial_{l} H_{k} \theta_{l}=-\frac{1}{2 r^{2}} \beta_{k i} \theta_{i}\left(-4 \gamma_{j k} \theta_{j}+2 \gamma_{m m} \theta_{k}\right)
$$

Note that the second term in the right hand side vanishes due to the fact that $\beta_{k i}=$ $-\beta_{i k}$, but not the first. To show this vanishes we proceed as follows. We have $d A_{R}=$ $R^{2} d A$ where $d A$ is the area element of the unit sphere $S$, so we can write $\int_{S_{R}} Z d A_{R}=$ $R^{2} \int_{S} Z d A$. Recall the identity

$$
\int_{S} \theta_{k} \theta_{l} d A=\frac{4 \pi}{3} \delta_{k l}
$$

This gives

$$
\int_{S_{R}} Z d A_{R}=-\frac{2 \pi}{3} \beta_{k i}\left(-4 \gamma_{j k} \delta_{i j}+2 \gamma_{m m} \delta_{i k}\right)=0
$$

due to the fact that $\beta_{k i}=-\beta_{i k}$. The integrand in the second term in (5.13) is

$$
-\frac{1}{2 r^{2}} \beta_{i k}\left(\gamma_{i j} \theta_{j}-\gamma_{m m} \theta_{i}\right) \theta_{k}
$$

which can be handled using the same method. It follows that

$$
\lim _{R \rightarrow \infty}\left(\int_{S_{R}} \xi^{i} \circ f \nabla_{k} V^{i} n^{k} d A_{h}-\int_{S_{R}} \nabla_{k}\left(\xi^{i} \circ f\right) n^{k} V_{i} d A_{h}\right)=0
$$

which completes the proof of the Lemma.

Lemma 5.6. For sufficiently small $G$, there is a constant $C$ such that the inequality

$$
\left|\int\left(\xi^{i} \circ f\right) L V_{i} d \mu_{h}\right| \leq C\left(\left\|h_{i j}-\delta_{i j}\right\|_{W_{\delta}^{2, p}}+\|\phi-\mathbf{i}\|_{W^{2, p}(\mathcal{B})}\right)|\xi|\|V\|_{W_{\delta-1}^{2, p}}
$$

holds, where for $\xi^{i}=\alpha_{i}+\beta^{i}{ }_{j} x^{j}$, we write $|\xi|=|\alpha|+|\beta|$.

Proof. Using Lemma 5.5 we consider

$$
\int L\left(\xi^{i} \circ f\right) V_{i} d \mu_{h}
$$

We need to estimate $\left\|L\left(\xi^{i} \circ f\right) V_{i}\right\|_{L^{1}}$. We expand out $L(\xi \circ f)$, dropping the reference to $f$ for brevity, and writing $\star$ to denote a general contraction

$$
L \xi=h \star \partial^{2} \xi+\Gamma \star \partial \xi+\partial \Gamma \star \xi+R \star \xi
$$

Using the form of $h_{i j}$ given in equation (5.5), we have

$$
\begin{aligned}
\Gamma & =O\left(1 / r^{2}\right)+v, & & \text { with } v \in L_{2 \delta-1}^{p} \\
\partial \Gamma & =O\left(1 / r^{3}\right)+z, & & \text { with } z \in L_{2 \delta-2}^{p} \\
R & =O\left(1 / r^{3}\right)+w, & & \text { with } w \in L_{2 \delta-2}^{p}
\end{aligned}
$$

Further, using the estimates from Lemma 5.2, the coefficients in the $O\left(1 / r^{2}\right)$ and $O\left(1 / r^{3}\right)$ terms can be estimated in terms of $|\gamma|$, where $\gamma_{i j}$ is the constant metric in (5.5), and the 
lower order terms can be estimated in terms of $h_{(2) i j}$. The term involving $\partial^{2} \xi$ has compact support and can be estimated in terms of $\phi-\mathbf{i}$. Multiplying $L \xi \circ f$ by $V$, using $\xi=$ $O(r)$ and $V \in W_{\delta-1}^{2, p}$, we find the result gives terms in $L_{\delta-3}^{p}$ and $L_{3 \delta-2}^{p}$ coming from the $O\left(1 / r^{2}\right), O\left(1 / r^{3}\right)$ terms and the Sobolev terms, respectively. Since $L_{3 \delta-2}^{p} \subset L_{\delta-3}^{p}$ and $L_{\delta-3}^{p} \subset L^{1}$, this means that using Lemma 5.2 we have an estimate of the form

$$
\begin{aligned}
\|L(\xi \circ f) V\|_{L^{1}} & \leq C\left(|\gamma|+\left\|h_{(2) i j}\right\|_{W_{2 \delta}^{2, p}}+\|\phi-\mathbf{i}\|_{W_{\mathcal{B}}^{2, p}}\right)|\xi|\|V\|_{W_{\delta-1}^{2, p}} \\
& \leq C\left(\left\|h_{i j}-\delta_{i j}\right\|_{W_{\delta}^{2, p}}+\|\phi-\mathbf{i}\|_{W^{2, p}(\mathcal{B})}\right)|\xi|\|V\|_{W_{\delta-1}^{2, p}}
\end{aligned}
$$

This completes the proof.

We now take, in the sense of distributions, the divergence of equation (5.2d), i.e. the equation

$$
G_{i j}-\left(\nabla_{(i} V_{j)}-\frac{1}{2} h_{i j} \nabla_{l} V^{l}\right)=8 \pi G\left(\Theta_{i j}-e^{U} \sigma_{i j} \chi_{f-1(\mathcal{B})}\right),
$$

which holds in $\mathbb{R}_{\mathcal{S}}^{3}$. For the first term on the left in equation (5.14) we use the Bianchi identity from Lemma2.1 to conclude $\nabla^{i} G_{i j}=0$ in the sense of distributions. In order to take the divergence of the second term on the left, note that it follows from the definition of $V$ that $V \in W_{\delta-1}^{1, p}$. The regularity assumptions on $h_{i j}$ imply that the identity

$$
\nabla^{j}\left(\nabla_{(i} V_{j)}-\frac{1}{2} h_{i j} \nabla_{l} V^{l}\right)=\frac{1}{2}\left(\Delta_{h} V_{i}+R_{i j} V^{j}\right)
$$

holds in the sense of distributions. For the first term on the right of (5.14), we use equation (5.2c). In the second term on the right in (5.14), using that the boundary condition 5.2b) is satisfied, we apply Lemma 2.2 to get the identity

$$
-\nabla^{j}\left(\Theta_{i j}-e^{U} \sigma_{i j} \chi_{f^{-1}(\mathcal{B})}\right)=\left[\nabla_{j}\left(e^{U} \sigma_{i}{ }^{j}\right)-e^{U}\left(n \epsilon-\sigma_{l}{ }^{l}\right) \partial_{i} U\right] \chi_{f^{-1}(\mathcal{B})}
$$

In view of the projected elastic equation (5.2a), this is equivalent to

$$
\begin{aligned}
-\nabla^{j}\left(\Theta_{i j}-e^{U} \sigma_{i j} \chi_{f^{-1}(\mathcal{B})}\right) & = \\
\left(\mathbb{I}_{f^{-1}(\mathcal{B})}\right. & \left.-\mathbb{P}_{f^{-1}(\mathcal{B})}\right)\left[\nabla_{j}\left(e^{U} \sigma_{i}{ }^{j}\right)-e^{U}\left(n \epsilon-\sigma_{l}{ }^{l}\right) \partial_{i} U\right] \chi_{f^{-1}(\mathcal{B})}
\end{aligned}
$$

where $\mathbb{I}_{f^{-1}(\mathcal{B})}$ is the identity operator in the space $L^{p}\left(f^{-1}(\mathcal{B})\right)$. Thus, defining the operator $L$ by

$$
L V_{i}=\Delta_{h} V_{i}+R_{i j} V^{j}
$$

we finally conclude that the equation

$$
L V_{i}=16 \pi G\left(\mathbb{I}_{f^{-1}(\mathcal{B})}-\mathbb{P}_{f^{-1}(\mathcal{B})}\right)\left[\nabla_{j}\left(e^{U} \sigma_{i}^{j}\right)-e^{U}\left(n \epsilon-\sigma_{l}^{l}\right) \partial_{i} U\right] \chi_{f^{-1}(\mathcal{B})}
$$

holds in $\mathbb{R}_{\mathcal{S}}^{3}$.

We observe that equation (5.15) implies that $L V$ is supported in $f^{-1}(\mathcal{B})$. Further, in view of the fact that $\mathbb{P}_{f^{-1}(\mathcal{B})}$ is a projection, we have

$$
\mathbb{P}_{f^{-1}(\mathcal{B})} L V=0 \text {. }
$$

Recall from section 4 that $\mathbb{P}_{\mathcal{B}}$ was defined in terms of the Killing fields $\xi^{i}(X)=\xi^{i}(\mathbf{i}(X))$ with $\xi^{i}(x)=\alpha^{i}+\beta^{i}{ }_{j} x^{j}$ for $\alpha^{i}, \beta^{i}{ }_{j}$ constants such that $\beta^{i}{ }_{j}=-\beta^{j}{ }_{i}$. It follows from the definition of $\mathbb{P}_{f^{-1}(\mathcal{B})}$, that we have

$$
0=\int_{\mathbb{R}_{\mathcal{S}}^{3}}\left(\xi^{i} \circ f(x)\right) \mathbb{P}_{f^{-1}(\mathcal{B})} z_{i} \chi_{f-1}(\mathcal{B}) d \mu_{h}
$$


for any $\xi^{i}$ of the above form and any $z_{i} \in L_{\mathrm{loc}}^{p}\left(\mathbb{R}_{\mathcal{S}}^{3}\right)$. Now define the linear mapping $\mathbb{Q}: L_{\delta-3}^{p}\left(\mathbb{R}_{\mathcal{S}}^{3}\right) \rightarrow \mathbb{R}^{6}$, by setting

$$
\mathbb{Q}_{\kappa}\left(z_{i}\right)=\int_{\mathbb{R}_{\mathcal{S}}^{3}}\left(\xi_{(\kappa)}^{i} \circ f\right) z_{i} d \mu_{h}, \quad \kappa=1, \ldots, 6
$$

where $\left\{\xi_{(\kappa)}\right\}_{\kappa=1}^{6}$ forms a basis for the space of Killing fields. We have the estimate

Lemma 5.7. There is a constant, depending on $f$ and $h_{i j}$ such that the inequality

$$
\|z\|_{L^{p}\left(f^{-1}(\mathcal{B})\right)} \leq C\left(\left\|\mathbb{P}_{f^{-1}(\mathcal{B})} z\right\|_{L^{p}\left(f^{-1}(\mathcal{B})\right)}+\left\|\mathbb{Q} z \chi_{f^{-1}(\mathcal{B})}\right\|_{\mathbb{R}^{6}}\right)
$$

holds.

Proof. From the definition of $\mathbb{P}_{f^{-1}(\mathcal{B})}$, we have

$$
z \chi_{f^{-1}(\mathcal{B})}=\mathbb{P}_{f^{-1}(\mathcal{B})} z \chi_{f^{-1}(\mathcal{B})}+n(\zeta \circ f) \chi_{f^{-1}(\mathcal{B})}
$$

where $\zeta$ is a Killing field, and from the definition of $\mathbb{Q}$, we have

$$
\begin{aligned}
\mathbb{Q}_{(\kappa)} z \chi_{f^{-1}(\mathcal{B})} & =\int_{\mathbb{R}_{\mathcal{S}}^{3}}\left(\xi_{(\kappa)}^{i} \circ f\right) n(\zeta \circ f) \chi_{f^{-1}(\mathcal{B})} d \mu_{h} \\
& =\int_{\mathcal{B}} \xi_{(\kappa)}^{i} \zeta_{i} d V
\end{aligned}
$$

for a basis of Killing fields $\left\{\xi_{(\kappa)}\right\}_{\kappa=1}^{6}$. In view of the non-degeneracy of the $L^{2}$ pairing on the space of Killing fields on $\mathcal{B}$, this means that there is a constant $C$ such that $\| n(\zeta \circ$ $f)\left\|_{L^{p}\left(f^{-1}(\mathcal{B})\right)} \leq C|| \mathbb{Q} n(\zeta \circ f) \chi_{f^{-1}(\mathcal{B})}\right\|_{\mathbb{R}^{6}}$. The inequality (5.17) now follows after an application of the triangle inequality.

Proposition 5.8. For sufficiently small $G$, there is a constant $C$ such that the estimate

$$
\|V\|_{W_{\delta-1}^{2, p}} \leq C\|\mathbb{Q} L V\|_{\mathbb{R}^{6}}
$$

holds.

Proof. Due to the boundedness of $\mathcal{B}$ and $f^{-1}(\mathcal{B})$, there is a constant $C$ such that

$$
\left\|z \chi_{f^{-1}(\mathcal{B})}\right\|_{L_{\delta-3}^{p}} \leq C\|z\|_{L^{p}\left(f^{-1}(\mathcal{B})\right)}
$$

Since $V$ solves (5.15), $L V$ is supported in $f^{-1}(\mathcal{B})$ so the above inequality applies to $L V$. Now making use of (5.16), the estimate (5.17), together with the estimate (5.8) from Lemma 5.4, we find that for $h_{i j}$ sufficiently close to $\delta_{i j}$, i.e. for $G$ suffiently close to 0 , the estimate (5.18) holds.

5.3. The main theorem. We are now able to prove

Theorem 5.9. For sufficiently small values of $G$, the solution to the reduced, projected, system of equations for a static, elastic, self-gravitating body, equations (5.2) is a solution to the full system (3.6) of equations for a static, elastic, self-gravitating body.

Proof. Recall the definition of $\mathbb{Q}$. We have

$$
\mathbb{Q}_{(\kappa)} L V=\int\left(\xi_{(\kappa)}^{i} \circ f\right) L V_{i} d \mu_{h}
$$

For small data, i.e. for sufficiently small $G$, $\xi$ is an approximate Killing field for $h_{i j}$. Therefore by Lemma 5.6, there is for sufficiently small $G$ a constant $C$ such that

$$
\|\mathbb{Q} L V\|_{\mathbb{R}^{6}} \leq C\left(\left\|h_{i j}-\delta_{i j}\right\|_{W_{\delta}^{2, p}}+\|\phi-\mathbf{i}\|_{W^{2, p}(\mathcal{B})}\right)\|V\|_{W_{\delta-1}^{2, p}}
$$


By (5.18), this implies that after possibly decreasing $G$, there is a constant $C$ such that

$$
\|V\|_{W_{\delta-1}^{2, p}} \leq C\left(\left\|h_{i j}-\delta_{i j}\right\|_{W_{\delta}^{2, p}}+\|\phi-\mathbf{i}\|_{W^{2, p}(\mathcal{B})}\right)\|V\|_{W_{\delta-1}^{2, p}}
$$

Hence, by Corollary 5.1 for sufficiently small values of $G$, we have

$$
\|V\|_{W_{\delta-1}^{2, p}} \leq \frac{1}{2}\|V\|_{W_{\delta-1}^{2, p}}
$$

Thus, in fact $V=0$. Hence equation (5.2d) implies the full Einstein equation (3.6c). Further, by (5.15) we have that

$$
\left(\mathbb{I}_{f^{-1}(\mathcal{B})}-\mathbb{P}_{f^{-1}(\mathcal{B})}\right)\left[\nabla_{j}\left(e^{U} \sigma_{i}{ }^{j}\right)-e^{U}\left(n \epsilon-\sigma_{l}{ }^{l}\right) \partial_{i} U\right] \chi_{f^{-1}(\mathcal{B})}=0
$$

while by equation $(\overline{5.2 \mathrm{a}})$,

$$
\mathbb{P}_{f^{-1}(\mathcal{B})}\left(\nabla_{j}\left(e^{U} \sigma_{i}{ }^{j}\right)-e^{U}\left(n \epsilon-\sigma_{l}{ }^{l}\right) \partial_{i} U\right)=0 \quad \text { in } f^{-1}(\mathcal{B})
$$

It follows that equation (3.6a) is satisfied, and hence that the solution $\left(\phi, U, h_{i j}\right)$ to the projected, reduced system, which was constructed using the implicit function theorem, is a solution to the full system (3.6).

\section{APPENDIX A.}

The way the spatial metric $h_{i j}$ is treated in section 4 may seem surprising at first. We start by reducing the Einstein equation (3.6c) using harmonic gauge, and then pull back the components of $h_{i j}$ in that gauge. Geometrically it might seem much more natural to start by pulling back the metric itself under $\hat{\phi}$. Doing this would result in replacing (3.6c) by

$$
\bar{G}_{A B}=8 \pi G\left(\bar{\Theta}_{A B}-e^{-\bar{U}_{\bar{\sigma}}} \bar{\sigma}_{A B} \chi_{\mathcal{B}}\right) \quad \text { in } \mathbb{R}_{\mathcal{B}}^{3},
$$

where $\bar{G}_{A B}$ is the Einstein tensor of $\bar{H}_{A B}$. Furthermore, we have that

$$
\bar{\Theta}_{A B}=\frac{1}{8 \pi G}\left[D_{A} \bar{U} D_{B} \bar{U}-\frac{1}{2} \bar{H}_{A B} \bar{H}^{C D} D_{C} \bar{U} D_{D} \bar{U}\right]
$$

and $\bar{\sigma}_{A B}$ is the extension of $\sigma_{A B}$. But then the quantity $\bar{\sigma}_{i}{ }^{A}$ in equation (3.16) depends on $\phi$ only through $\widehat{\psi}^{A}$, since the remaining factors in

$$
\bar{\sigma}_{i}^{A}=\widehat{\psi}^{B}{ }_{i} J \bar{\sigma}_{B C} \bar{H}^{A C}
$$

depend only on $\bar{H}_{A B}$. Let us now look at the nature of Eq. (3.13), when $\left(\phi, \bar{U}, \bar{H}_{A B}\right)$ are used as the basic variables rather than $\left(\phi, \bar{U}, \bar{h}_{i j}\right)$. There are potentially two terms depending on second derivatives of $\phi$. One comes from taking a derivative of $\widehat{\psi}^{A}{ }_{i}$ in equation (A.1) above. The other comes from writing the Christoffel symbols of $h_{i j}$ entering the left hand side of (3.13) in terms of $\phi$. Remarkably, both these terms cancel! For the linearization of the residual of equation (3.13) at $\left(\phi=\mathbf{i}, \bar{U}=0, \bar{H}_{A B}=\delta_{A B}\right)$ the only surviving term will involve first partial derivatives of $\bar{H}_{A B}$, i.e., terms which break diffeomorphism invariance on $\mathbb{R}_{\mathcal{B}}^{3}$. However note that the very presence of the a priori given domain $\mathcal{B}$ (which of course is the reason why this whole manoeuvre was performed) breaks diffeomorphism invariance. It is thus no wonder, that, after going over to the material picture in the sense of using the full pull-back of $h_{i j}$, the elastic equation gives us terms which break diffeomorphism invariance. 


\section{APPENDIX B.}

The following Newtonian version of the argument in this paper was contributed by the anonymous referee. In the Newtonian theory the equations, with the standard definition of the stress, taking the specific mass to be 1 , are

$$
\begin{aligned}
\nabla_{j} \sigma_{i}{ }^{j}+n \partial_{i} U & =0 \quad \text { in } f^{-1}(\mathcal{B}), \\
\left.\sigma_{i}{ }^{j} n_{j}\right|_{\partial f^{-1}(\mathcal{B})} & =0, \\
\Delta U & =4 \pi G n \chi_{f^{-1}(\mathcal{B})} \quad \text { in } \mathbb{R}_{\mathcal{S}}^{3}
\end{aligned}
$$

The argument to show that the projected version of (B.1a) implies (B.1a) would go as follows. We define $X_{i}$ to be the solution of

$$
\Delta X_{i}=\nabla_{j} \sigma_{i}{ }^{j}+n \partial_{i} U
$$

tending to zero at infinity. Since

$$
\rho_{i}=\nabla_{j} \sigma_{i}^{j}+n \partial_{i} U
$$

the right hand side of (B.2), has compact support, $X_{i}$ has a multipole expansion in a neighborhood of infinity,

$$
-4 \pi X_{i}=\frac{M_{i}}{r}+\frac{x^{j} D_{i j}}{r^{3}}+O\left(r^{-3}\right)
$$

Here

$$
\begin{aligned}
M_{i} & =\int_{R^{3}} \rho_{i}(x) d^{3} x \\
D_{i j} & =\int_{R^{3}} x^{j} \rho_{i}(x) d^{3} x .
\end{aligned}
$$

Writing

$$
n \partial_{i} U=\nabla_{j} \Theta_{i}{ }^{j}
$$

where $\Theta_{i}{ }^{j}=\Theta_{i j}$ is given by (3.7) with $h_{i j}$ replaced by $\delta_{i j}$, using the fact that $\Theta_{i j}=$ $O\left(r^{-4}\right)$ at infinity, and taking into account $(\mathrm{B} .1 \mathrm{~b})$, we obtain

$$
\begin{aligned}
M & =0 \\
D_{i j} & =-\int_{\mathbb{R}^{3}}\left(\sigma_{i}{ }^{j}+\Theta_{i}{ }^{j}\right) d^{3} x
\end{aligned}
$$

Thus,

$$
X_{i}=-\frac{D_{i j} x^{j}}{4 \pi r^{3}}+O\left(r^{-3}\right),
$$

where $D_{i j}=D_{j i}$, in agreement with (5.9). Lemma 5.5 then applies with $\Delta$ in the role of $L$ and Lemma 5.6 applies with $\delta_{i j}$ in the role of $h_{i j}$. Proposition 5.8 also applies. The argument of the proof of Theorem 5.9 then applies to show that $X_{i}=0$, therefore equation (B.1a) holds. 
Acknowledgements. We are grateful to the anonymous referee for reading the paper carefully and suggesting important improvements. We also thank him for providing the material presented in appendix $\mathrm{A}$

The Newton Institute, where part of the work was done, provided support and hospitality for which we are grateful. RB thanks the Albert Einstein Institute for its hospitality. BS would like to thank the members of the geometric analysis group at the AEI for helpful discussions in the initial stages of this work. LA was supported in part by the National Science Foundation Grant DMS-0407732. RB was supported by Fonds zur Förderung der Wissenschaftlichen Forschung, Project Nr. P16745-N02

\section{REFERENCES}

[1] Adams, R. A. Sobolev spaces. Academic Press, New York-London, 1975. Pure and Applied Mathematics, Vol. 65.

[2] Agmon, S.; Douglis, A.; Nirenberg, L. Estimates near the boundary for solutions of elliptic partial differential equations satisfying general boundary conditions. II. Comm. Pure Appl. Math. 17 (1964), 35-92.

[3] Bartnik, R. The mass of an asymptotically flat manifold. Comm. Pure Appl. Math. 39 (1986), no. 5, 661-693.

[4] Beig, R.; Schmidt, B. G. Relativistic elasticity. Classical Quantum Gravity 20 (2003), no. 5, 889-904.

[5] Beig, R.; Schmidt, B. G. Static, self-gravitating elastic bodies. R. Soc. Lond. Proc. Ser. A Math. Phys. Eng. Sci. 459 (2003), no. 2029, 109-115.

[6] Beig, R.; Schmidt, B. G. Relativistic elastostatics. I. Bodies in rigid rotation. Classical Quantum Gravity 22 (2005), no. 11, 2249-2268.

[7] Beig, R.; Simon, W. The stationary gravitational field near spatial infinity. Gen. Relativity Gravitation 12 (1980), no. 12, 1003-1013 (1981).

[8] Beig, R.; Simon, W. On the spherical symmetry of static perfect fluids in general relativity. Lett. Math. Phys. 21 (1991), no. 3, 245-250.

[9] Beig, R.; Wernig-Pichler, M. On the motion of a compact elastic body. gr-qc/0508025. 2005. To appear in Comm. Math. Phys.

[10] Burenkov, V. I. Sobolev spaces on domains, Teubner-Texte zur Mathematik, vol. 137. B. G. Teubner Verlagsgesellschaft mbH, Stuttgart, 1998.

[11] Carter, B.; Quintana, H. Foundations of general relativistic high-pressure elasticity theory. Proc. Roy. Soc. London Ser. A 331 (1972), 57-83.

[12] Christodoulou, D. On the geometry and dynamics of crystalline continua. Ann. Inst. H. Poincaré Phys. Théor. 69 (1998), no. 3, 335-358.

[13] Christodoulou, D. The action principle and partial differential equations, Annals of Mathematics Studies, vol. 146. Princeton University Press, Princeton, NJ, 2000.

[14] Gilbarg, D.; Trudinger, N. S. Elliptic partial differential equations of second order, Grundlehren der Mathematischen Wissenschaften, vol. 224. Springer-Verlag, Berlin, 2nd ed., 1983.

[15] Heilig, U. On the existence of rotating stars in general relativity. Comm. Math. Phys. 166 (1995), no. 3, 457-493.

[16] Herglotz, G. Über die Mechanik des deformierbaren Körpers vom Standpunkte der Relativitätsteorie. Annalen der Physik 36 (1911), 493-533.

[17] Kennefick, D.; Ó Murchadha, N. Weakly decaying asymptotically flat static and stationary solutions to the Einstein equations. Classical Quantum Gravity 12 (1995), no. 1, 149-158.

[18] Kijowski, J.; Magli, G. Relativistic elastomechanics as a Lagrangian field theory. J. Geom. Phys. 9 (1992), no. 3, 207-223.

[19] Kijowski, J.; Magli, G. Unconstrained variational principle and canonical structure for relativistic elasticity. Rep. Math. Phys. 39 (1997), no. 1, 99-112.

[20] Lang, S. Differential manifolds. Springer-Verlag, New York, 2nd ed., 1985.

[21] Le Dret, H. Structure of the set of equilibrated loads in nonlinear elasticity and applications to existence and nonexistence. J. Elasticity 17 (1987), no. 2, 123-141.

[22] Liechtenstein, L. Gleichgewicthsfiguren rotirende flüssigkeiten. Springer, Berlin, 1933.

[23] Lindblad, H. Well posedness for the motion of a compressible liquid with free surface boundary. Comm. Math. Phys. 260 (2005), no. 2, 319-392.

[24] Lindblad, H. Well-posedness for the motion of an incompressible liquid with free surface boundary. Ann. of Math. (2) 162 (2005), no. 1, 109-194.

[25] Lindblom, L. Stationary stars are axisymmetric. Astrophys. J. 208 (1976), no. 3, part 1, 873-880. 
[26] Marsden, J. E.; Hughes, T. J. R. Mathematical foundations of elasticity. Dover Publications Inc., New York, 1994. Corrected reprint of the 1983 original.

[27] Rayner, C. B. Elasticity in general relativity. Proc. Roy. Soc. Ser. A 272 (1963), 44-53.

[28] Rendall, A. D. Theorems on existence and global dynamics for the Einstein equations. Living Rev. Relativ. 5 (2002), 2002-6, 62 pp. (electronic).

[29] Stoppelli, F. Un teorema di esistenza ed unicità relativo alle equazioni dell'elastostatica isoterma per deformazioni finite. Ricerche Mat. 3 (1954), 247-267.

[30] Valent, T. Boundary value problems of finite elasticity, Springer Tracts in Natural Philosophy, vol. 31. Springer-Verlag, New York, 1988.

E-mail address: larsa@math.miami.edu

Albert Einstein Institute, Am MÜhlenberg 1, D-14476 Potsdam, Germany and Department of Mathematics, University of Miami, Coral Gables, FL 33124, USA

E-mail address: robert.beig@univie.ac.at

Physics Faculty/Gravitational Physics, University of Vienna, BoltzManngasse 5, A-1090 VIENNA, AUSTRIA

E-mail address: bernd@aei.mpg.de

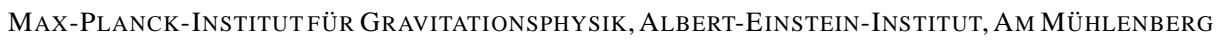
1, D-14476 GOLM, GERMANY 